SLAC-PUB-9281

July 2002

\title{
Perspectives on Exclusive Processes in QCD"̈
}

\author{
Stanley J. Brodsky \\ Stanford Linear Accelerator Center, Stanford University \\ Stanford, California 94309 \\ e-mail: sjbth@slac.stanford.edu
}

\begin{abstract}
Hard hadronic exclusive processes are now at the forefront of QCD studies, particularly because of their role in the interpretation of exclusive hadronic $B$ decays, processes which are essential for determining the CKM phases and the physics of $C P$ violation. Perturbative QCD and its factorization properties at high momentum transfer provide an essential guide to the phenomenology of exclusive amplitudes at large momentum transfer - the leading power fall-off of form factors and fixed-angle cross sections, the dominant helicity structures, and their color transparency properties. The reduced amplitude formalism provides an extension of the perturbative QCD predictions to exclusive nuclear amplitudes. The hard scattering subprocess $T_{H}$ controlling the leading-twist amplitude is only evaluated in the QCD perturbative domain where the propagator virtualities are above the separation scale. A critical question is the momentum transfer required such that leading-twist perturbative QCD contributions dominate. I review some of the contentious theoretical issues and empirical challenges to Perturbative QCD based analyses, such as the magnitude of the leading-twist contributions, the role of soft and higher twist QCD mechanisms, the effects of non-zero orbital angular momentum, the possibility of single-spin asymmetries in deeply virtual Compton scattering, the role of hidden color in nuclear wavefunctions, the behavior of the ratio of Pauli and Dirac nucleon form factors, the origin of anomalous $J / \psi$ decays, the apparent breakdown of color transparency in quasi-elastic proton-proton scattering, and the measurement of hadron and photon wavefunctions in diffractive dijet production.
\end{abstract}

Invited Talk, presented at the

Workshop on Exclusive Processes at High Momentum Transfer

Jefferson Lab, Newport News, Virginia

May 15-18, 2002

\footnotetext{
${ }^{*}$ Work supported by the Department of Energy, contract DE-AC03-76SF00515.
} 


\section{Introduction}

Exclusive processes provide a unique window for viewing QCD processes and hadron dynamics at the amplitude level[1]. Hadronic exclusive processes are closely related to exclusive hadronic $B$ decays, processes which are essential for determining the CKM phases and the physics of $C P$ violation. The universal light-front wavefunctions which control hard exclusive processes such as form factors, deeply virtual Compton scattering, high momentum transfer photoproduction, and two-photon processes, are also required for computing exclusive heavy hadron decays 2, 3, 4, 5], such as $B \rightarrow K \pi, B \rightarrow \ell \nu \pi$, and $B \rightarrow K p \bar{p}$ [6]. The same physics issues, including color transparency, hadron helicity rules, and the question of dominance of leading-twist perturbative QCD mechanisms enter in both realms of physics. New tests of theory and comprehensive measurements of hard exclusive amplitudes can be carried out for electroproduction at Jefferson Laboratory and in two-photon collisions at CLEO, Belle, and BaBar [7]. The perturbative QCD approach to exclusive processes is now facing a number of strong empirical challenges. New data from Jefferson Laboratory [8] for the ratio of Pauli and Dirac form factors of the proton appears to be at variance with QCD expectations. This has led to a new focus on the range of validity of leading-twist perturbative QCD predictions and the necessity to have better theoretical control on higher-twist contributions. The Pauli form factor is particularly interesting, since it measures spin-orbit $\vec{S} \cdot \vec{L}$ couplings and thus the presence of orbital angular momentum in the proton light-front wavefunction. The new Jefferson Laboratory results appear to call into question hadron helicity conservation [9, 10, a key feature of the leading-twist predictions. It is often claimed that the leading-twist predictions for the spacelike pion and proton form factors strongly underestimate their empirical magnitudes. The assumed relation between diffractive dijet production to the shape of the projectile light-front wavefunction has also been questioned. I will

give my perspective on these challenges to theory in this report. QCD mechanisms for exclusive processes are illustrated in Figs. 1 and 2.

\section{Perturbative QCD and Exclusive Processes}

There has been considerable progress analyzing exclusive and diffractive reactions at large momentum transfer from first principles in QCD. Rigorous statements can be made on the basis of asymptotic freedom and factorization theorems which separate the underlying hard quark and gluon subprocess amplitude from the nonperturbative physics of the hadronic wavefunctions. The leading-power contribution to exclusive hadronic amplitudes such as quarkonium decay, heavy hadron decay, and scattering amplitudes where hadrons are scattered with large momentum transfer can often be factorized as a convolution of distribution amplitudes $\phi_{H}\left(x_{i}, \Lambda\right)$ and hard-scattering 
(a) Light Cone Fock Expansion

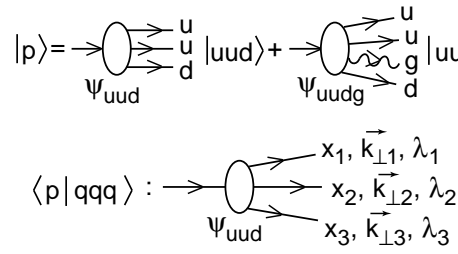

$$
\begin{aligned}
& \psi_{n}\left(x_{i}, \vec{k}_{\perp i}, \lambda_{i}\right): \sum_{i=1}^{n} x_{i}=1, \sum_{i=1}^{n} \vec{k}_{\perp i}=0
\end{aligned}
$$

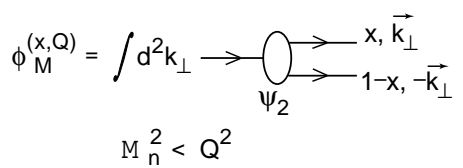

(c) Deep Inelastic $l p \rightarrow l^{\prime} X\left\langle p\left|J^{+}(z) J^{+}(0)\right| p\right\rangle$

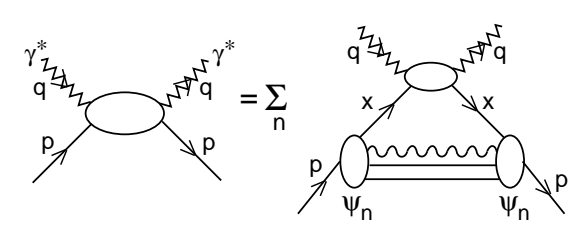

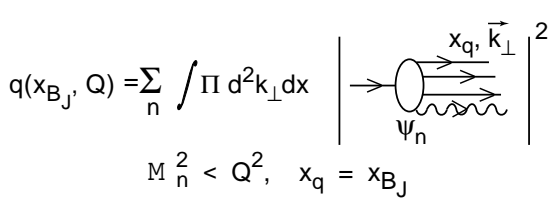

${ }_{8348 \mathrm{~A} 10}^{9-97}$ (d)

Form Factors $l p \rightarrow l^{\prime} p^{\prime}\left\langle p^{\prime} \lambda{ }^{\prime}\left|J^{+}(0)\right| p \lambda\right\rangle$
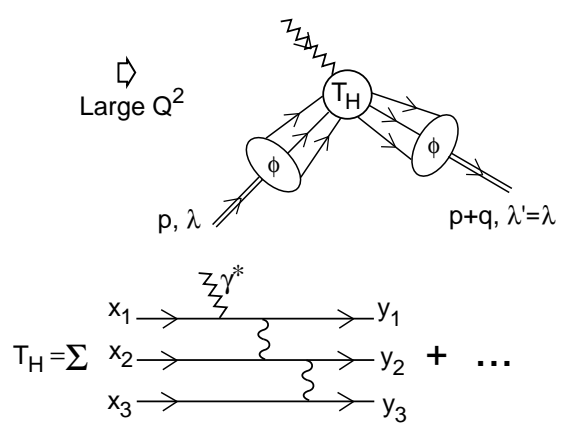

$=\frac{\alpha_{S}^{2}}{Q^{4}} f\left(x_{i}, y_{i},\right)$

(e) Compton $\gamma p \rightarrow \gamma^{\prime} p^{\prime}\left\langle p^{\prime}\left|J^{\mu}(z) J^{\nu}(0)\right| p\right\rangle$
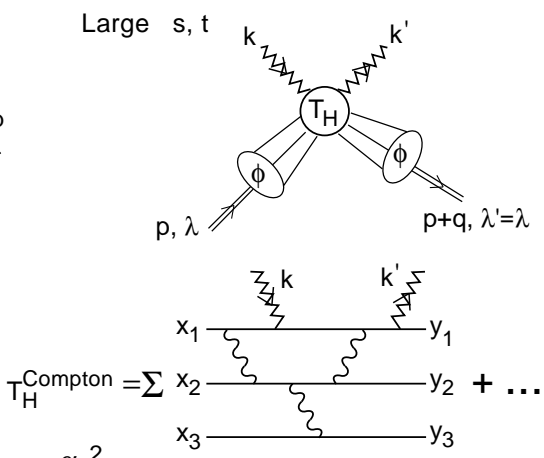

$=\frac{\alpha_{s}^{2}}{P_{T}^{4}} f\left(x_{i}, y_{i}, \theta_{c m}\right)$

Figure 1: Representation of QCD hadronic processes in the light-cone Fock expansion. (a) The valence uud and $u u d g$ contributions to the light-cone Fock expansion for the proton. (b) The distribution amplitude $\phi(x, Q)$ of a meson expressed as an integral over its valence light-cone wavefunction restricted to $q \bar{q}$ invariant mass less than $Q$. (c) Representation of deep inelastic scattering and the quark distributions $q(x, Q)$ as probabilistic measures of the light-cone Fock wavefunctions. The sum is over the Fock states with invariant mass less than $Q$. (d) Exact representation of spacelike form factors of the proton in the light-cone Fock basis. The sum is over all Fock components. At large momentum transfer the leading-twist contribution factorizes as the product of the hard scattering amplitude $T_{H}$ for the scattering of the valence quarks collinear with the initial to final direction convoluted with the proton distribution amplitude. (e) Leading-twist factorization of the Compton amplitude at large momentum transfer. 

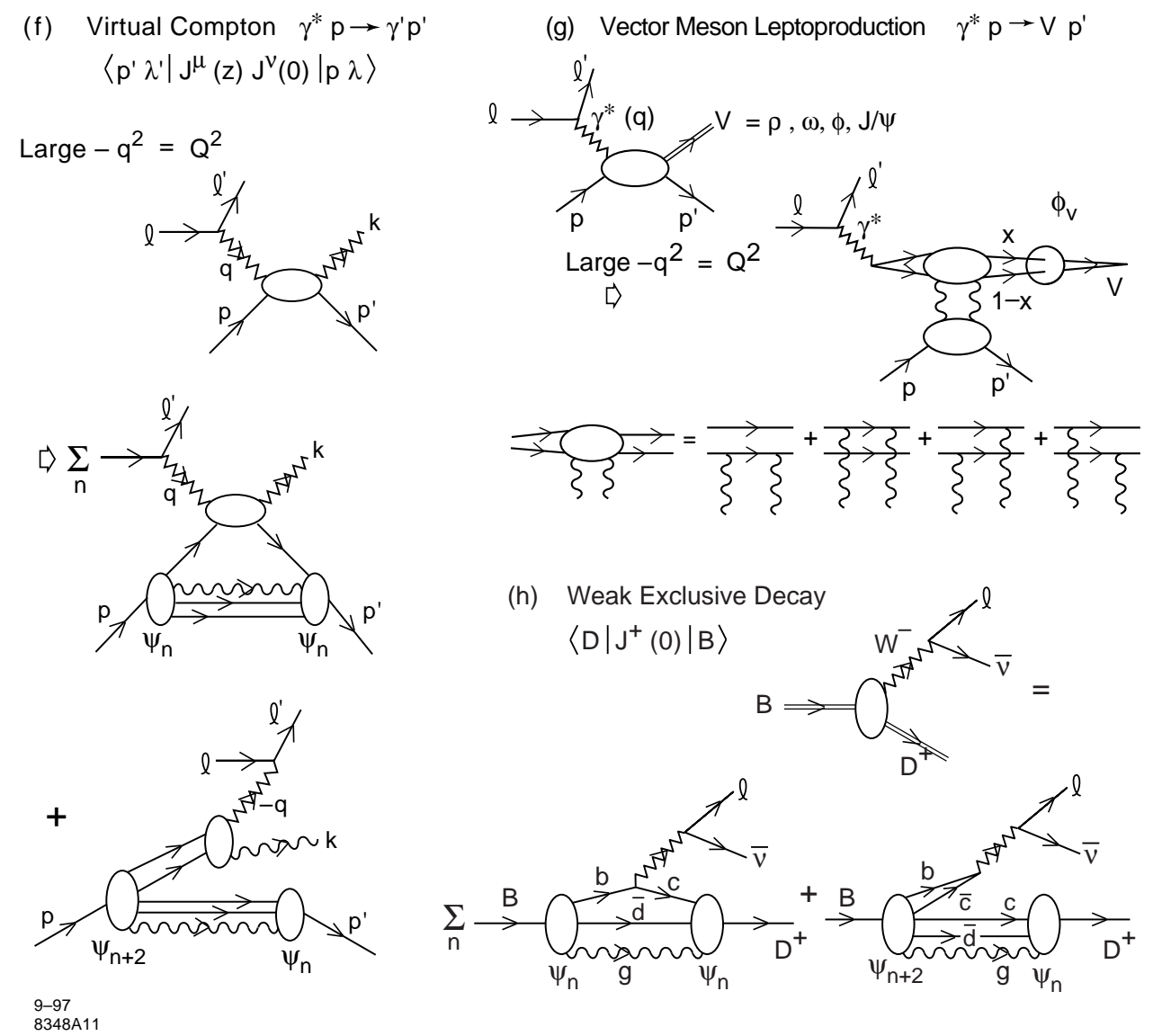

(h) Weak Exclusive Decay

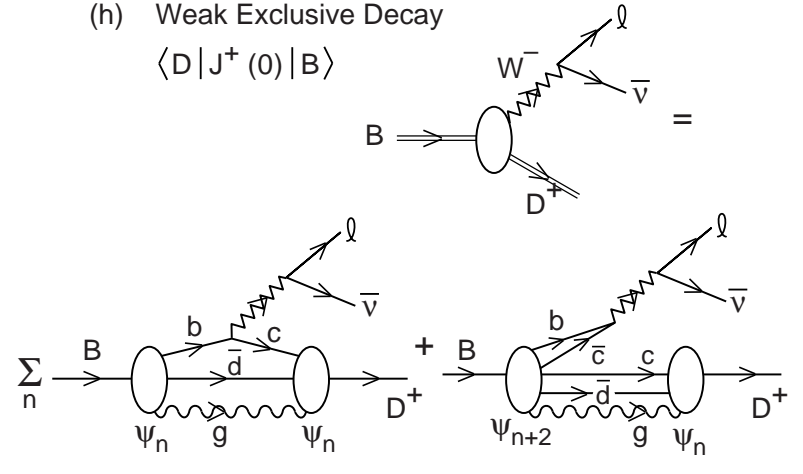

Figure 2: (f) Representation of deeply virtual Compton scattering in the lightcone Fock expansion at leading twist. Both diagonal $n \rightarrow n$ and off-diagonal $n+$ $2 \rightarrow n$ contributions are required. (g) Diffractive vector meson production at large photon virtuality $Q^{2}$ and longitudinal polarization. The high energy behavior involves two gluons in the $t$ channel coupling to the compact color dipole structure of the upper vertex. The bound-state structure of the vector meson enters through its distribution amplitude. (h) Exact representation of the weak semileptonic decays of heavy hadrons in the light-cone Fock expansion. Both diagonal $n \rightarrow n$ and offdiagonal pair annihilation $n+2 \rightarrow n$ contributions are required. 
quark/gluon scattering amplitudes $T_{H}$ integrated over the light-cone momentum fractions of the valence quarks 11]:

$$
\mathcal{M}_{\text {Hadron }}=\int \prod \phi_{H}^{(\Lambda)}\left(x_{i}, \lambda_{i}\right) T_{H}^{(\Lambda)} d x_{i}
$$

Here $T_{H}^{(\Lambda)}$ is the underlying quark-gluon subprocess scattering amplitude in which each incident and final hadron is replaced by valence quarks with collinear momenta $k_{i}^{+}=x_{i} p_{H}^{+}, \vec{k}_{\perp i}=x_{i} \vec{p}_{\perp H}$. The invariant mass of all intermediate states in $T_{H}$ is evaluated above the separation scale $\mathcal{M}_{n}^{2}>\Lambda^{2}$. The essential part of the hadronic wavefunction is the distribution amplitude [11], defined as the integral over transverse momenta of the valence (lowest particle number) Fock wavefunction; e.g. for the pion

$$
\phi_{\pi}\left(x_{i}, Q\right) \equiv \int d^{2} k_{\perp} \psi_{q \bar{q} / \pi}^{(Q)}\left(x_{i}, \vec{k}_{\perp i}, \lambda\right)
$$

where the separation scale $\Lambda$ can be taken to be order of the characteristic momentum transfer $Q$ in the process. It should be emphasized that the hard scattering amplitude $T_{H}$ is evaluated in the QCD perturbative domain where the propagator virtualities are above the separation scale.

The leading power fall-off of the hard scattering amplitude as given by dimensional counting rules follows from the nominal scaling of the hard-scattering amplitude: $T_{H} \sim 1 / Q^{n-4}$, where $n$ is the total number of fields (quarks, leptons, or gauge fields) participating in the hard scattering 12, 13. Thus the reaction is dominated by subprocesses and Fock states involving the minimum number of interacting fields. In the case of $2 \rightarrow 2$ scattering amplitudes, this implies $\frac{d \sigma}{d t}(A B \rightarrow C D)=F_{A B \rightarrow C D}(t / s) / s^{n-2}$. In the case of form factors, the dominant helicity conserving amplitude obeys $F(t) \sim$ $(1 / t)^{n_{H}-1}$ where $n_{H}$ is the minimum number of fields in the hadron $H$. The full predictions from PQCD modify the nominal scaling by logarithms from the running coupling and the evolution of the distribution amplitudes. In some cases, such as large angle $p p \rightarrow p p$ scattering, there can be "pinch" contributions 14 when the scattering can occur from a sequence of independent near-on shell quark-quark scattering amplitudes at the same CM angle. After inclusion of Sudakov suppression form factors, these contributions also have a scaling behavior close to that predicted by constituent counting.

The constituent counting rules 12, 13] were originally derived in 1973 before the development of QCD, in anticipation that the underlying theory of hadron physics would be renormalizable and close to a conformal theory. The factorizable structure of hard exclusive amplitudes in terms of a convolution of valence hadron wavefunctions times a hard scattering quark scattering amplitude was also proposed. Upon the discovery of the asymptotic freedom in QCD, there was a systematical development of the theory of hard exclusive reactions, including factorization theorems, counting rules, and evolution equations for the hadronic distribution amplitudes [15, 16, 17, 18. In a remarkable recent development, Polchinski and Strassler 19 have derived 
the constituent counting rules using string duality, mapping features of gravitational theories in higher dimensions $\left(A d S_{5}\right)$ to physical QCD in ordinary $3+1$ space-time.

The distribution amplitudes which control leading-twist exclusive amplitudes at high momentum transfer can be related to the gauge-invariant Bethe-Salpeter wavefunction at equal light-cone time $\tau=x^{+}$. The logarithmic evolution of the hadron distribution amplitudes $\phi_{H}\left(x_{i}, Q\right)$ with respect to the resolution scale $Q$ can be derived from the perturbatively-computable tail of the valence light-cone wavefunction in the high transverse momentum regime. The DGLAP evolution of quark and gluon distributions can also be derived in an analogous way by computing the variation of the Fock expansion with respect to the separation scale. Other key features of the perturbative QCD analyses are: (a) evolution equations for distribution amplitudes which incorporate the operator product expansion, renormalization group invariance, and conformal symmetry 11, 20, 21, 22, 23]; (b) hadron helicity conservation which follows from the underlying chiral structure of QCD [9]; (c) color transparency, which eliminates corrections to hard exclusive amplitudes from initial and final state interactions at leading power and reflects the underlying gauge theoretic basis for the strong interactions 24 and (d) hidden color degrees of freedom in nuclear wavefunctions, which reflect the color structure of hadron and nuclear wavefunctions 25]. There have also been recent advances eliminating renormalization scale ambiguities in hard-scattering amplitudes via commensurate scale relations 26] which connect the couplings entering exclusive amplitudes to the $\alpha_{V}$ coupling which controls the QCD heavy quark potential.

\section{The Pion Form Factor}

The pion spacelike form factor provides an important illustration of the perturbative QCD formalism. The proof of factorization begins with the exact Drell-Yan-West representation [27, 28, 29] of the current in terms of the light-cone Fock wavefunctions (see Section 7.) The integration over the momenta of the constituents of each wavefunction can be divided into two domains $\mathcal{M}_{n}^{2}<\Lambda^{2}$ and $\mathcal{M}_{n}^{2}>\Lambda^{2}$, where $\mathcal{M}_{n}^{2}$ is the invariant mass of the $n$-particle state. $\Lambda$ plays the role of a separation scale. In practice, it can be taken to be of order of the momentum transfer.

Consider the contribution of the two-particle Fock state. The argument of the final state pion wavefunction is $k_{\perp}+(1-x) q_{\perp}$. First take $k_{\perp}$ small. At high momentum transfer where

$$
\mathcal{M}^{2} \sim \frac{(1-x)^{2} q_{\perp}^{2}}{x(1-x)}=\frac{Q^{2}(1-x)}{x}>\Lambda^{2},
$$

one can iterate the equation of motion for the valence light-front wavefunction using the one gluon exchange kernel. Including all of the hard scattering domains, one can organize the result into the factorized form:

$$
F_{\pi}\left(Q^{2}\right)=\int_{0}^{1} d x \int_{0}^{1} d y \phi_{\pi}(y, \Lambda) T_{H}\left(x, y, Q^{2}\right) \phi_{\pi}(x, \Lambda)
$$


where $T_{H}$ is the hard-scattering amplitude $\gamma^{*}(q \bar{q}) \rightarrow(q \bar{q})$ for the production of the valence quarks nearly collinear with each meson, and $\phi_{M}(x, \Lambda)$ is the distribution amplitude for finding the valence $q$ and $\bar{q}$ with light-cone fractions of the meson's momentum, integrated over invariant mass up to $\Lambda$. The process independent distribution amplitudes contain the soft physics intrinsic to the nonperturbative structure of the hadrons. Note that $T_{H}$ is non-zero only if $\frac{(1-x) Q^{2}}{x}>\Lambda^{2}$ and $\frac{(1-y) Q^{2}}{y}>\Lambda^{2}$. In this hard-scattering domain, the transverse momenta in the formula for $\underline{T}_{H}$ can be ignored at leading power, so that the structure of the process has the form of hard scattering on collinear quark and gluon constituents: $T_{H}\left(x, y, Q^{2}\right)=\frac{16 \pi C_{F} \alpha_{s}\left(Q^{* 2}\right)}{(1-x)(1-y) Q^{2}}\left(1+\mathcal{O}\left(\alpha_{s}\right)\right)$ and thus 115, 16, 17, 11, 18, 30, 31, 32, 33.

$$
F_{\pi}\left(Q^{2}\right)=\frac{16 \pi C_{F} \alpha_{s}\left(Q^{* 2}\right)}{Q^{2}} \int_{0}^{\widehat{x}} d x \frac{\phi_{\pi}(x, \Lambda)}{(1-x)} \int_{0}^{\widehat{y}} d y \frac{\phi_{\pi}(y, \Lambda)}{(1-y)},
$$

to leading order in $\alpha_{s}\left(Q^{* 2}\right)$ and leading power in $1 / Q$. Here $C_{F}=4 / 3$ and $Q^{*}$ can be taken as the BLM scale [34]. The endpoint regions of integration $1-x<\frac{\Lambda^{2}}{Q^{2}}=1-\widehat{x}$ and $1-y<\frac{\Lambda^{2}}{Q^{2}}=1-\widehat{y}$ are to be explicitly excluded in the leading-twist formula. However, since the integrals over $x$ and $y$ are convergent, one can formally extend the integration range to $0<x<1$ and $0<y<1$ with an error of higher twist. This is only done for convenience - the actual domain only encompasses the off-shell regime. The contribution from the endpoint regions of integration, $x \sim 1$ and $y \sim 1$, are power-law and Sudakov suppressed and thus contribute corrections at higher order in $1 / Q$ [16, 17, 11]. The contributions from non-valence Fock states and corrections from fixed transverse momentum entering the hard subprocess amplitude are higher twist, i.e., power-law suppressed. Loop corrections involving hard momenta give next-to-leading-order (NLO) corrections in $\alpha_{s}$.

It is sometimes assumed that higher twist terms in the $\mathrm{LC}$ wave function, such as those with $L_{z} \neq 0$, have flat distributions at the $x \rightarrow 0,1$ endpoints. This is difficult to justify since it would correspond to bound state wavefunctions which fall-off in transverse momentum but have no fall-off at large $k_{z}$. After evolution to $Q^{2} \rightarrow \infty$, higher twist distributions can evolve eventually to constant behavior at $x=0,1$; however, the wavefunctions are in practice only being probed at moderate scales. In fact, if the higher twist terms are evaluated in the soft domain, then there is no evolution at all. A recent analysis by Beneke 35 indicates that the $1 / Q^{4}$ contribution to the pion form factor is only logarithmically enhanced even if the twist-3 term is flat at the endpoints. It is also possible that contributions from the twist three $q \bar{q} g$ light-front wavefunctions may well cancel even this enhancement.

Thus perturbative QCD can unambiguously predict the leading-twist behavior of exclusive amplitudes. These contributions only involve the truncated integration domain of $x$ and $k_{\perp}$ momenta where the quark and gluon propagators and couplings are perturbative; by definition the soft regime is excluded. The central question is then whether the PQCD leading-twist prediction can account for the observed 
leading power-law fall-off of the form factors and other exclusive processes. Assuming the pion distribution amplitude is close to its asymptotic form, one can predict the normalization of exclusive amplitudes such as the spacelike pion form factor $Q^{2} F_{\pi}\left(Q^{2}\right)$. Next-to-leading order predictions are available which incorporate higher order corrections to the pion distribution amplitude as well as the hard scattering amplitude[21, 36, 37, 38]. The natural renormalization scheme for the QCD coupling in hard exclusive processes is $\alpha_{V}(Q)$, the effective charge defined from the scattering of two infinitely-heavy quark test charges. Assuming $\alpha_{V}\left(Q^{*}\right) \simeq 0.4$ at the BLM scale $Q^{*}$, the QCD LO prediction appears to be smaller by approximately a factor of 2 compared to the presently available data extracted from pion electroproduction experiments [34]. However, the extrapolation from spacelike $t$ to the pion pole in electroproduction may be unreliable, in the same sense that lattice gauge theory extrapolations to $m_{\pi}^{2} \rightarrow 0$ are known to be nonanalytic. Thus it is not clear that there is an actual discrepancy between perturbative QCD and experiment. It would be interesting to develop predictions for the transition form factor $F_{q \bar{q} \rightarrow \pi}\left(t, q^{2}\right)$ that is in effect measured in electroproduction.

Compton scattering is a key test of the perturbative QCD approach 39, 40, 41]. A detailed recalculation of the helicity amplitudes and differential cross section for proton Compton scattering at fixed angle has been carried out recently by Brooks and Dixon 41] at leading-twist and at leading order in $\alpha_{s}$. They use contour deformations to evaluate the singular integrals in the light-cone momentum fractions arising from pinch contributions. The shapes and scaling behavior predicted by perturbative QCD agree well with the existing data [42]. In order to reduce uncertainties associated with $\alpha_{s}$ and the three-quark wave function normalization, Brooks and Dixon have normalized the Compton cross section using the proton elastic form factor. The theoretical predictions for the ratio of Compton scattering to electron-proton scattering is about an order of magnitude below existing experimental data. However, this discrepancy of a factor of 3 in the relative normalization of the amplitudes could be attributed to the fact that the number of diagrams contributing to the Compton amplitude at next-to-leading order $\left(\alpha_{s}^{3}\right)$ is much larger in Compton scattering compared to the proton form factor.

A debate has continued on whether processes such as the pion and proton form factors and elastic Compton scattering $\gamma p \rightarrow \gamma p$ might be dominated by higher twist mechanisms until very large momentum transfers [43, 44, 45, 46, 47]. For example, if one assumes that the light-cone wavefunction of the pion has the form $\psi_{\text {soft }}\left(x, k_{\perp}\right)=$ $A \exp \left(-b \frac{k_{\perp}^{2}}{x(1-x)}\right)$, then the Feynman endpoint contribution to the overlap integral at small $k_{\perp}$ and $x \simeq 1$ will dominate the form factor compared to the hard-scattering contribution until very large $Q^{2}$. However, this form for $\psi_{\text {soft }}\left(x, k_{\perp}\right)$ does not falloff at all for $k_{\perp}=0$ and $k_{z} \rightarrow-\infty$. A soft QCD wavefunction would be expected to be exponentially suppressed in this regime, as in the BHL model $\psi_{n}^{\text {soft }}\left(x_{i}, k_{\perp i}\right)=$ $A \exp \left(-b \sum_{i}^{n}\left[\frac{\vec{k}_{\perp}^{2}+m^{2}}{x}\right]_{i}\right)$ 48. The endpoint contributions are also suppressed by a 
QCD Sudakov form factor 49, reflecting the fact that a near-on-shell quark must radiate if it absorbs large momentum. If the endpoint contribution dominates proton Compton scattering, then both photons will interact on the same quark line in a local fashion and the amplitude is real, in strong contrast to the QCD predictions which have a complex phase structure. The perturbative QCD predictions 39, 40, 41] for the Compton amplitude phase can be tested in virtual Compton scattering by interference with Bethe-Heitler processes [50]. Recently the "handbag" approach to Compton scattering [46, 47] has been applied to $\bar{p} p \rightarrow \gamma \gamma$ at large energy [51]. In this case, one assumes that the process occurs via the exchange of a diquark with light-cone momentum fraction $x \sim 0$, so that the hard subprocess is $\bar{q} q \rightarrow \gamma \gamma$ where the quarks annihilate with the full energy of the baryons and nearly on-shell. The critical question is whether the proton wavefunction has significant support when the massive diquark has zero light-front momentum fraction, since the diquark light-cone kinetic energy and the bound state wavefunction become far-off shell $k_{F}^{2} \sim-\left(m^{2}+k_{\perp}^{2}\right) / x \rightarrow-\infty$ in this domain.

It is interesting to compare the calculation of a meson form factors in QCD with the calculations of form factors of bound states in QED. The analog to a soft wave-

function is the Schrödinger-Coulomb solution $\psi_{1 s}(\vec{k}) \propto\left(1+\vec{p}^{2} /\left(\alpha m_{\text {red }}\right)^{2}\right)^{-2}$, and the full wavefunction, which incorporates transversely polarized photon exchange, differs by a factor $\left(1+\vec{p}^{2} / m_{\text {red }}^{2}\right)$. Thus the leading-twist dominance of form factors in QED occurs at relativistic scales $Q^{2}>m_{\text {red }}^{2}$ 近.

\section{Perturbative QCD Calculation of Baryon Form Factors}

The baryon form factor at large momentum transfer provides another important example of the application of perturbative QCD to exclusive processes. Away from possible special points in the $x_{i}$ integrations (which are suppressed by Sudakov form factors) baryon form factors can be written to leading order in $1 / Q^{2}$ as a convolution of a connected hard-scattering amplitude $T_{H}$ convoluted with the baryon distribution amplitudes. The $Q^{2}$-evolution of the baryon distribution amplitude can be derived from the operator product expansion of three quark fields or from the gluon exchange kernel. Taking into account the evolution of the baryon distribution amplitude, the nucleon magnetic form factors at large $Q^{2}$, has the form [11, 17, 9]

$$
G_{M}\left(Q^{2}\right) \rightarrow \frac{\alpha_{s}^{2}\left(Q^{2}\right)}{Q^{4}} \sum_{n, m} b_{n m}\left(\log \frac{Q^{2}}{\Lambda^{2}}\right)^{\gamma_{n}^{B}+\gamma_{n}^{B}}\left[1+\mathcal{O}\left(\alpha_{s}\left(Q^{2}\right), \frac{m^{2}}{Q^{2}}\right)\right]
$$

where the $\gamma_{n}^{B}$ are computable anomalous dimensions 52 of the baryon three-quark wave function at short distance, and the $b_{m n}$ are determined from the value of the distribution amplitude $\phi_{B}\left(x, Q_{0}^{2}\right)$ at a given point $Q_{0}^{2}$ and the normalization of $T_{H}$. 
Asymptotically, the dominant term has the minimum anomalous dimension. The contribution from the endpoint regions of integration, $x \sim 1$ and $y \sim 1$, at finite $k_{\perp}$ is Sudakov suppressed [16, 17, 11]; however, the endpoint region may play a significant role in phenomenology.

The proton form factor appears to scale at $Q^{2}>5 \mathrm{GeV}^{2}$ according to the PQCD predictions. See Fig. 3. Nucleon form factors are approximately described phenomenologically by the well-known dipole form $G_{M}\left(Q^{2}\right) \simeq 1 /\left(1+Q^{2} / 0.71 \mathrm{GeV}^{2}\right)^{2}$ which behaves asymptotically as $G_{M}\left(Q^{2}\right) \simeq\left(1 / Q^{4}\right)\left(1-1.42 \mathrm{GeV}^{2} / Q^{2}+\cdots\right)$. This suggests that the corrections to leading twist in the proton form factor and similar exclusive processes involving protons become important in the range $Q^{2}<1.4 \mathrm{GeV}^{2}$.

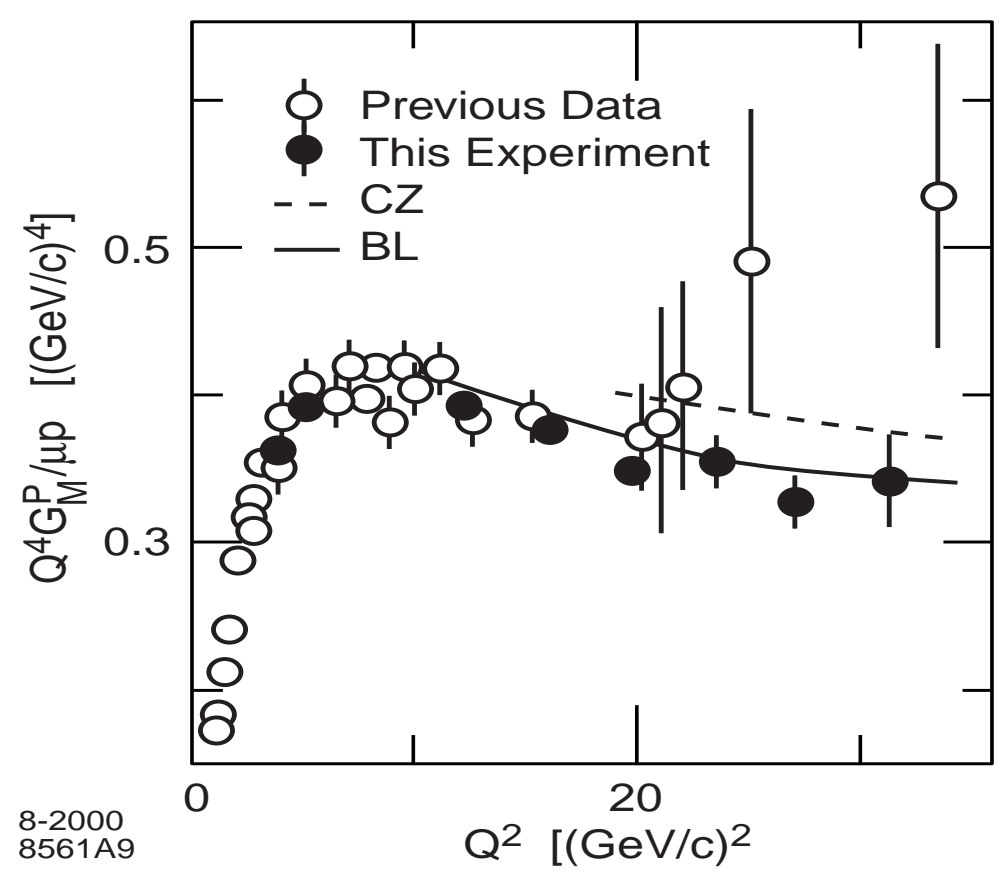

Figure 3: Predictions for the normalization and sign of the proton form factor at high $Q^{2}$ using perturbative QCD factorization and QCD sum rule predictions for the proton distribution amplitude (From Ji et al. [53) The curve labelled BL has arbitrary normalization and incorporates the fall-off of two powers of the running coupling. The dotted line is the QCD sum rule prediction of given by Chernyak and Zhitnitsky [54, 55]. The results are similar for the model distribution amplitudes of King and Sachrajda 56, and Gari and Stefanis 57.

The shape of the distribution amplitude controls the normalization of the leadingtwist prediction for the proton form factor. If one assumes that the proton distribution amplitude has the asymptotic form: $\phi_{N}=C x_{1} x_{2} x_{3}$, then the convolution with the 
leading order form for $T_{H}$ gives zero! If one takes a non-relativistic form peaked at $x_{i}=1 / 3$, the sign is negative, requiring a crossing point zero in the form factor at some finite $Q^{2}$. The broad asymmetric distribution amplitudes advocated by Chernyak and Zhitnitsky [54, 55] gives a more satisfactory result. If one assumes a constant value of $\alpha_{s}=0.3$, and $f_{N}=5.3 \times 10^{-3} \mathrm{GeV}^{2}$, the leading order prediction is below the data by a factor of $\approx 3$. However, since the form factor is proportional to $\alpha_{s}^{2} f_{N}^{2}$, one can obtain agreement with experiment by a simple renormalization of the parameters. For example, if one uses the central value of Ioffe's determination $f_{N}=8 \times 10^{-3} \mathrm{GeV}^{2}$, then good agreement is obtained[58]. The normalization of the proton distribution amplitude is also important for estimating the proton decay rate [59]. The most recent lattice results [60] suggest a significantly larger normalization for the required proton matrix elements, 3 to 5 times larger than earlier phenomenological estimates. One can also use PQCD to predict ratios of various baryon and isobar form factors assuming isospin or $S U(3)$-flavor symmetry for the basic wave function structure. Results for the neutral weak and charged weak form factors assuming standard $S U(2) \times U(1)$ symmetry can also be derived [61].

A useful technique for obtaining the solutions to the baryon evolution equations is to construct completely antisymmetric representations as a polynomial orthonormal basis for the distribution amplitude of multi-quark bound states. In this way one obtain a distinctive classification of nucleon $(N)$ and Delta $(\Delta)$ wave functions and the corresponding $Q^{2}$ dependence which discriminates $N$ and $\Delta$ form factors. More recently Braun and collaborators have shown how one can use conformal symmetry to classify the eigensolutions of the baryon distribution amplitude[23]. They identify a new 'hidden' quantum number which distinguishes components in the $\lambda=3 / 2$ distribution amplitudes with different scale dependence. They are able to find analytic solution of the evolution equation for $\lambda=3 / 2$ and $\lambda=1 / 2$ baryons where the two lowest anomalous dimensions for the $\lambda=1 / 2$ operators (one for each parity) are separated from the rest of the spectrum by a finite 'mass gap'. These special states can be interpreted as baryons with scalar diquarks. Their results may support Carlson's solution [62] to the puzzle that the proton to $\Delta$ form factor falls faster [63] than other $p \rightarrow N^{*}$ amplitudes if the $\Delta$ distribution amplitude has a symmetric $x_{1} x_{2} x_{3}$ form.

In a remarkable new development, Pobylitsa et al. 64 have shown how to compute transition form factors linking the proton to nucleon-pion states which have minimal invariant mass $W$. A new soft pion theorem for high momentum transfers allows one to compute the three quark distribution amplitudes for the near threshold pion states from a chiral rotation. The new soft pion results are in a good agreement with the SLAC electroproduction data for $W^{2}<1.4 \mathrm{GeV}^{2}$ and $7<Q^{2}<30.7 \mathrm{GeV}^{2}$.

\section{Hadron Helicity Conservation}

Hadron helicity conservation (HHC) is a QCD selection rule concerning the behavior

of helicity amplitudes at high momentum transfer, such as fixed CM scattering. Since 
the convolution of $T_{H}$ with the light-cone wavefunctions projects out states with $L_{z}=0$, the leading hadron amplitudes conserve hadron helicity [9, 10]. Thus the dominant amplitudes are those in which the sum of hadron helicities in the initial state equals the sum of hadron helicities in the final state; other helicity amplitudes are relatively suppressed by an inverse power in the momentum transfer.

In the case of electron-proton scattering, hadron helicity conservation states that the proton helicity-conserving form factor ( which is proportional to $G_{M}$ ) dominates over the proton helicity-flip amplitude (proportional to $G_{E} / \sqrt{\tau}$ ) at large momentum transfer. Here $\tau=Q^{2} / 4 M^{2}, Q^{2}=-q^{2}$. Thus HHC predicts $G_{E}\left(Q^{2}\right) / \sqrt{\tau} G_{M}\left(Q^{2}\right) \rightarrow 0$ at large $Q^{2}$. The new data from Jefferson Laboratory [8] which shows a decrease in the ratio $G_{E}\left(Q^{2}\right) / G_{M}\left(Q^{2}\right)$ is not itself in disagreement with the HHC prediction.

The leading-twist QCD motivated form $Q^{4} G_{M}\left(Q^{2}\right) \simeq$ const $/ Q^{4} \ln Q^{2} \Lambda^{2}$ provides a good guide to both the time-like and spacelike proton form factor data at $Q^{2}>5 \mathrm{GeV}^{2}$ [65]. However, the Jefferson Laboratory data 8] appears to suggest $Q F_{2}\left(Q^{2}\right) / F_{1}\left(Q^{2}\right) \simeq$ const, for the ratio of the proton's Pauli and Dirac form factors in contrast to the nominal expectation $Q^{2} F_{2}\left(Q^{2}\right) / F_{1}\left(Q^{2}\right) \simeq$ const expected (modulo logarithms) from PQCD. It should however be emphasized that a PQCD-motivated fit is not precluded. For example, Hiller, Hwang and I [66] have noted that the form

$$
\frac{F_{2}\left(Q^{2}\right)}{F_{1}\left(Q^{2}\right)}=\frac{\mu_{A}}{1+\left(Q^{2} / c\right) \ln ^{b}\left(1+Q^{2} / a\right)}
$$

with $\mu_{A}=1.79, a=4 m_{\pi}^{2}=0.073 \mathrm{GeV}^{2}, b=-0.5922, c=0.9599 \mathrm{GeV}^{2}$ also fits the data well. The extra logarithmic factor is not unexpected for higher twist contributions. This fit is shown in Fig. 4 . The fitted form is consistent with hadron helicity conservation. The predictions for the time-like domain using simple crossing of the above form is shown by the dotted lines.

The study of time-like hadronic form factors using $e^{+} e^{-}$colliding beams can provide very sensitive tests of HHC, since the virtual photon in $e^{+} e^{-} \rightarrow \gamma^{*} \rightarrow h_{A} \bar{h}_{B}$ always has spin \pm 1 along the beam axis at high energies. Angular momentum conservation implies that the virtual photon can "decay" with one of only two possible angular distributions in the center of momentum frame: $\left(1+\cos ^{2} \theta\right)$ for $\left|\lambda_{A}-\lambda_{B}\right|=1$ and $\sin ^{2} \theta$ for $\left|\lambda_{A}-\lambda_{B}\right|=0$ where $\lambda_{A}$ and $\lambda_{B}$ are the helicities of the outgoing hadrons. Hadronic helicity conservation, as required by QCD, greatly restricts the possibilities. It implies that $\lambda_{A}+\lambda_{B}=0$. Consequently, angular momentum conservation requires $\left|\lambda_{A}\right|=\left|\lambda_{B}\right|=l / 2$ for baryons, and $\left|\lambda_{A}\right|=\left|\lambda_{B}\right|=0$ for mesons; thus the angular distributions for any sets of hadron pairs are now completely determined at leading twist: $\frac{d \sigma}{d \cos \theta}\left(e^{+} e^{-}=B \bar{B}\right) \propto 1+\cos ^{2} \theta$ and $\frac{d \sigma}{d \cos \theta}\left(e^{+} e^{-}=M \bar{M}\right) \propto \sin ^{2} \theta$. Verifying these angular distributions for vector mesons and other higher spin mesons and baryons would verify the vector nature of the gluon in QCD and the validity of PQCD applications to exclusive reactions.

It is usually assumed that a heavy quarkonium state such as the $J / \psi$ always 

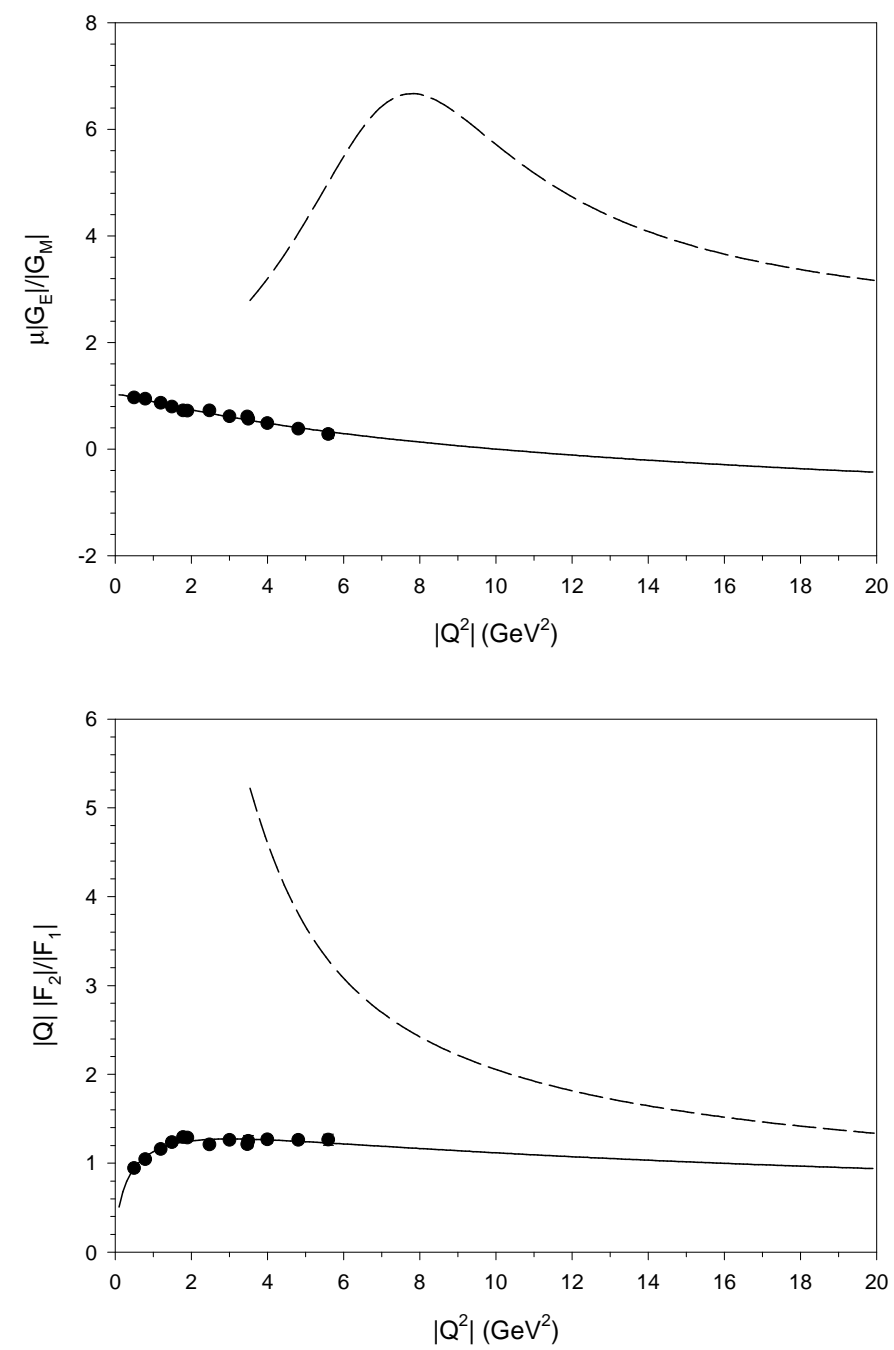

Figure 4: Perturbative QCD motivated fit [66] to the spacelike form factor ratios and $G_{E} / G_{M}$ and $Q F_{2} / F_{1}$. The fit is described in the text. The data are from Jefferson Laboratory [8]. Predictions for the time-like form factor ratios are shown as dotted curves. 
decays to light hadrons via the annihilation of its heavy quark constituents to gluons. However, as Karliner and I [67] have shown, the transition $J / \psi \rightarrow \rho \pi$ can also occur by the rearrangement of the $c \bar{c}$ from the $J / \psi$ into the $|q \bar{q} c \bar{c}\rangle$ intrinsic charm Fock state of the $\rho$ or $\pi$. On the other hand, the overlap rearrangement integral in the decay $\psi^{\prime} \rightarrow \rho \pi$ will be suppressed since the intrinsic charm Fock state radial wavefunction of the light hadrons will evidently not have nodes in its radial wavefunction. This observation provides a natural explanation of the long-standing puzzle why the $J / \psi$ decays prominently to two-body pseudoscalar-vector final states in conflict with HHC, whereas the $\psi^{\prime}$ does not. If the intrinsic charm explanation is correct, then this mechanism will complicate the analysis of virtually all heavy hadron decays such as $J / \psi \rightarrow p \bar{p}$. In addition, the existence of intrinsic charm Fock states, even at a few percent level, provides new, competitive decay mechanisms for $B$ decays which are nominally CKM-suppressed 68]. For example, the weak decays of the B-meson to twobody exclusive states consisting of strange plus light hadrons, such as $B \rightarrow \pi K$, are expected to be dominated by penguin contributions since the tree-level $b \rightarrow s u \bar{u}$ decay is CKM suppressed. However, higher Fock states in the B wave function containing charm quark pairs can mediate the decay via a CKM-favored $b \rightarrow s c \bar{c}$ tree-level transition. The presence of intrinsic charm in the $b$ meson can be checked by the observation of final states containing three charmed quarks, such as $B \rightarrow J / \psi D \pi$ [69.

\section{Other Applications}

There are a large number of measured exclusive reactions in which the empirical power law fall-off predicted by dimensional counting and PQCD appears to be accurate over a large range of momentum transfer. The approach to scaling of $s^{7} d \sigma / d t\left(\gamma p \rightarrow \pi^{+} n\right)$

shown in Fig. 5 appears to indicate that leading-twist PQCD is applicable at momentum transfers exceeding a few $\mathrm{GeV}$. If anything, the scaling appears to work too well, considering that one expects logarithmic deviations from the running of the QCD coupling and logarithmic evolution of the hadron distribution amplitudes. The deviations from scaling at lower energies [71] are interesting and can be attributed to $s$-channel resonances or perhaps heavy quark threshold effects, merging into the fixed-angle scaling in a similar way as one observes the approach to leading-twist Bjorken-scaling behavior in deep inelastic scattering via quark-hadron duality[72]. The absence of significant corrections to leading-twist scaling suggests that the running coupling is effectively frozen at the kinematics relevant to the data. If higher-twist soft processes are conspiring to mimic leading-twist scaling $s^{7} d \sigma / d t\left(\gamma p \rightarrow \pi^{+} n\right)$, then we would have the strange situation of seeing two separate kinematic domains of $s^{7}$ scaling of the photoproduction cross section. It has been argued 44, 73] that the Compton amplitude is dominated by soft end-point contributions of the proton wavefunctions where the two photons both interact on a quark line carrying nearly all of the pro- 


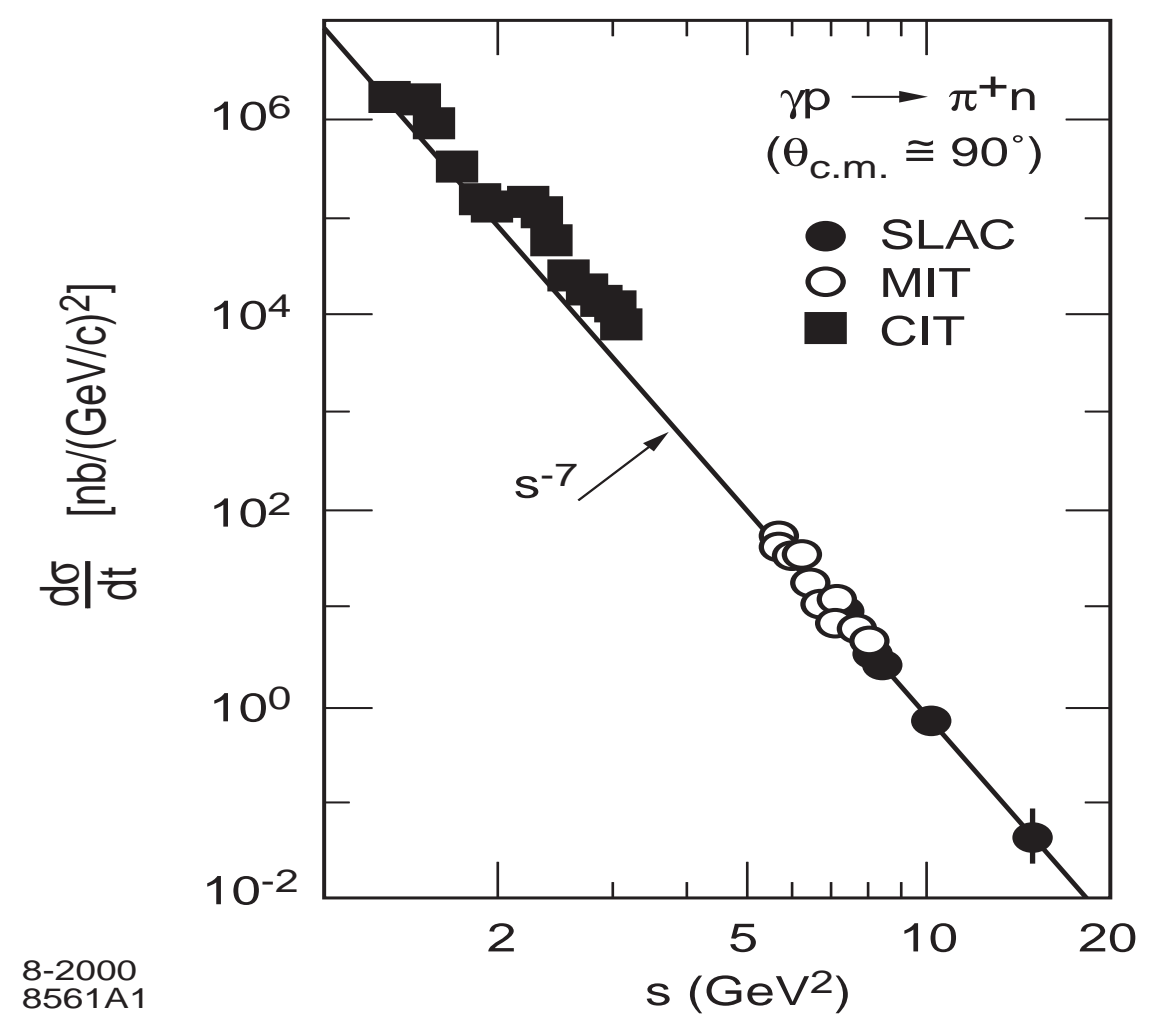

Figure 5: Comparison of photoproduction data with the dimensional counting powerlaw prediction. The data are summarized in Anderson et al. [70]

ton's momentum. However, a corresponding soft endpoint explanation of the observed $s^{7} d \sigma / d t\left(\gamma p \rightarrow \pi^{+} n\right)$ scaling of the pion photoproduction data is not apparent; there is no endpoint contribution which could explain the success of dimensional counting in large-angle pion photoproduction apparent in Fig. 5 .

Exclusive two-photon processes where two photons annihilate into hadron pairs $\gamma \gamma \rightarrow H \bar{H}$ at high transverse momentum provide highly valuable probes of coherent effects in quantum chromodynamics. For example, in the case of exclusive final states at high momentum transfer and fixed $\theta_{c m}$ such as $\gamma \gamma \rightarrow p \bar{p}$ or meson pairs, photonphoton collisions provide a time-like microscope for testing fundamental scaling laws of PQCD and for measuring distribution amplitudes. Counting rules predict asymptotic fall-off $s^{4} d \sigma / d t \sim f(t / s)$ for meson pairs and $s^{6} d \sigma / d t \sim f(t / s)$ for baryon pairs. Hadron-helicity conservation predicts dominance of final states with $\lambda_{H}+\lambda_{\bar{H}}=0$. The angular dependence reflects the distribution amplitudes. One can also study $\gamma^{*} \gamma \rightarrow$ hadron pairs in $e^{ \pm} e^{-}$collisions as a function of photon virtuality, the time-like analog of deeply virtual Compton scattering which is sensitive to the two hadron distribution amplitude. One can also study the interference of the time-like Compton amplitude with the bremsstrahlung amplitude $e^{ \pm} e \rightarrow B B e^{ \pm} e^{-}$. where a time-like 
photon produces the pair. The $e^{ \pm}$asymmetry measures the relative phase of the time-like hadron form factor with that of the virtual Compton amplitude.

The PQCD predictions for the two-photon production of charged pions and kaons is insensitive to the shape of the meson distribution amplitudes. In fact, the ratio of the $\gamma \gamma \rightarrow \pi^{+} \pi^{-}$and $e^{+} e^{-} \rightarrow \mu^{+} \mu^{-}$amplitudes at large $s$ and fixed $\theta_{C M}$ can be predicted since the ratio is nearly insensitive to the running coupling and the shape of the pion distribution amplitude:

$$
\frac{\frac{d \sigma}{d t}\left(\gamma \gamma \rightarrow \pi^{+} \pi^{-}\right)}{\frac{d \sigma}{d t}\left(\gamma \gamma \rightarrow \mu^{+} \mu^{-}\right)} \sim \frac{4\left|F_{\pi}(s)\right|^{2}}{1-\cos ^{2} \theta_{\text {c.m. }}}
$$

The comparison of the PQCD prediction for the sum of $\pi^{+} \pi^{-}$plus $K^{+} K^{-}$channels with recent CLEO data [76] is shown in Fig. 6. Results for separate pion and kaon channels have been given by the TPC $/ 2 \gamma$ collaboration [74]. The angular distribution

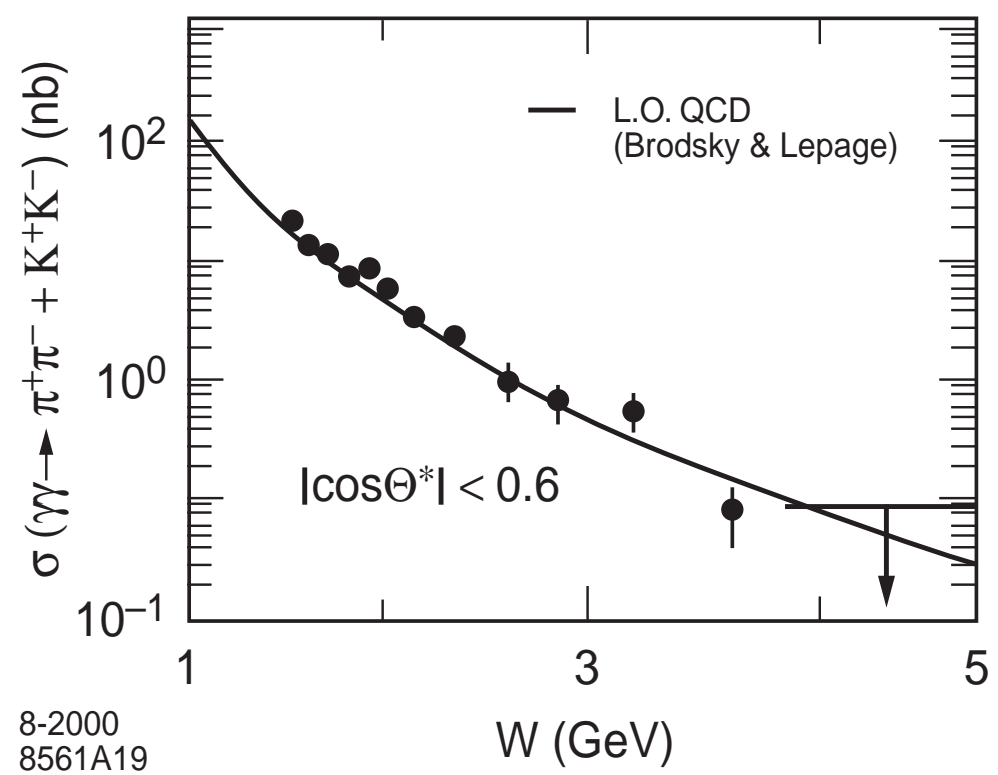

Figure 6: Comparison of the sum of $\gamma \gamma \rightarrow \pi^{+} \pi^{-}$and $\gamma \gamma \rightarrow K^{+} K^{-}$meson pair production cross sections with the perturbative QCD prediction 75 normalized to the timelike pion form factor. The data are from the CLEO collaboration 76.

of meson pairs is also predicted by PQCD at large momentum transfer. The CLEO data for charged pion and kaon pairs show a clear transition to the angular distribution predicted by PQCD for $W=\sqrt{s}_{\gamma \gamma}>2 \mathrm{GeV}$. Similarly in $\gamma \gamma \rightarrow p \bar{p}$ one can see a dramatic change in the fixed angle distribution as one enters the hard scattering domain. It is clearly important to measure the two-photon production of neutral pions and $\rho^{+} \rho^{-}$cross sections in view of their strong sensitivity to the shape of 
meson distribution amplitudes. Furthermore, the ratio of $\pi^{+} \pi^{-}$to $\pi^{0} \pi^{0}$ cross sections is highly sensitive to the production dynamics. The ratio $\frac{\sigma\left(\gamma \gamma \rightarrow \pi^{0} \pi^{0}\right)}{\sigma\left(\gamma \gamma \rightarrow \pi^{+} \pi^{-}\right)}$at fixed angles which is very small in PQCD, and of order 1 in soft handbag models.

An interesting contribution to $K^{+} p \rightarrow K^{+} p$ scattering comes from the exchange of the common $u$ quark. The quark interchange amplitude for $A+B \rightarrow C+D$ can be written as a convolution of the four light-cone wavefunctions multiplied by a factor $\Delta^{-}=P_{A}^{-}+P_{B}^{-}-\sum_{i} k_{i}^{-}$, the inverse of the central propagator 77 . The interchange amplitude is consistent with constituent counting rule scaling, and often provides a phenomenologically accurate representation of the $\theta_{c . m}$. angular distribution at large momentum transfer. For example, the angular distribution of processes such as $K^{+} p \rightarrow K^{+} p$ appear to follow the predictions based on hard scattering diagrams based on quark interchange, e.g., $T_{H}\left(\left(u_{1} \bar{s}\right)\left(u_{2} u_{3} d\right) \rightarrow\left(u_{2} \bar{s}\right)\left(u_{1} u_{3} d\right)\right.$ [77]. This mechanism also provides constraints on Regge intercepts $\alpha_{R}(t)$ for meson exchange trajectories at large momentum transfer [78]. An extensive review of this phenomenology is given in the review by Sivers et al. 779.

One of the most interesting areas of exclusive processes is to amplitudes where the nuclear wavefunction has to absorb large momentum transfer. For example, the helicity-conserving deuteron form factor is predicted to scale as $F_{d}\left(Q^{2}\right) \propto\left(Q^{2}\right)^{-5}$ reflecting the minimal six quark component of nuclear wavefunction. The deuteron form factor at high $Q^{2}$ is sensitive to wavefunction configurations where all six quarks overlap within an impact separation $b_{\perp i}<\mathcal{O}(1 / Q)$. The leading power-law fall off predicted by QCD is $F_{d}\left(Q^{2}\right)=f\left(\alpha_{s}\left(Q^{2}\right)\right) /\left(Q^{2}\right)^{5}$, where, asymptotically 80, 25], $f\left(\alpha_{s}\left(Q^{2}\right)\right) \propto \alpha_{s}\left(Q^{2}\right)^{5+2 \gamma}$. In general, the six-quark wavefunction of a deuteron is a mixture of five different color-singlet states. The dominant color configuration at large distances corresponds to the usual proton-neutron bound state. However, at small impact space separation, all five Fock color-singlet components eventually acquire equal weight, i.e., the deuteron wavefunction evolves to $80 \%$ "hidden color" 225]. The relatively large normalization of the deuteron form factor observed at large $Q^{2}$ hints at sizable hidden-color contributions 81. Hidden color components can also play a predominant role in the reaction $\gamma d \rightarrow J / \psi p n$ at threshold if it is dominated by the multi-fusion process $\gamma g g \rightarrow J / \psi$. In the case of nuclear structure functions beyond the single nucleon kinematic limit, $1<x_{b j}<A$, the nuclear light-cone momentum must be transferred to a single quark, requiring quark-quark correlations between quarks of different nucleons in a compact, far-off-shell regime. This physics is also sensitive to the part of the nuclear wavefunction which contains hidden-color components in distinction from a convolution of separate color-singlet nucleon wavefunctions. One also sees the onset of the predicted perturbative QCD scaling behavior for exclusive nuclear amplitudes such as deuteron photodisintegration $(n=1+6+3+3=13)$ $s^{11} \frac{d \sigma}{d t}(\gamma d \rightarrow p n) \sim$ constant at fixed CM angle. The measured deuteron form factor and the deuteron photodisintegration cross section appear to follow the leading-twist QCD predictions at large momentum transfers in the few $\mathrm{GeV}$ region [82, 83]. To first approximation, the proton and neutron share the deuteron's momentum equally. 
Since the deuteron form factor contains the probability amplitudes for the proton and neutron to scatter from $p / 2$ to $p / 2+q / 2$; it is natural to define the reduced deuteron form factor 80 , 25]

$$
f_{d}\left(Q^{2}\right) \equiv \frac{F_{d}\left(Q^{2}\right)}{F_{1 N}\left(\frac{Q^{2}}{4}\right) F_{1 N}\left(\frac{Q^{2}}{4}\right)} .
$$

The effect of nucleon compositeness is removed from the reduced form factor. QCD then predicts the scaling

$$
f_{d}\left(Q^{2}\right) \sim \frac{1}{Q^{2}}
$$

i.e. the same scaling law as a meson form factor. This scaling is consistent with experiment for $Q \gtrsim 1 \mathrm{GeV}$. In the case of deuteron photodisintegration $\gamma d \rightarrow p n$ the amplitude requires the scattering of each nucleon at $t_{N}=t_{d} / 4$. The perturbative QCD scaling is 84

$$
\frac{d \sigma}{d \Omega_{c . m .}}(\gamma d \rightarrow n p)=\frac{1}{\sqrt{s\left(s-M_{d}^{2}\right)}} \frac{F_{n}^{2}\left(t_{d} / 4\right) F_{p}^{2}\left(t_{d} / 4\right) f_{r e d}^{2}\left(\theta_{c . m}\right)}{p_{\perp}^{2}} .
$$

The predicted scaling of the reduced photodisintegration amplitude $f_{\text {red }}\left(\theta_{\text {c.m. }}\right) \simeq$ const is also consistent with experiment 84, 82, 83]. See Fig. 7.

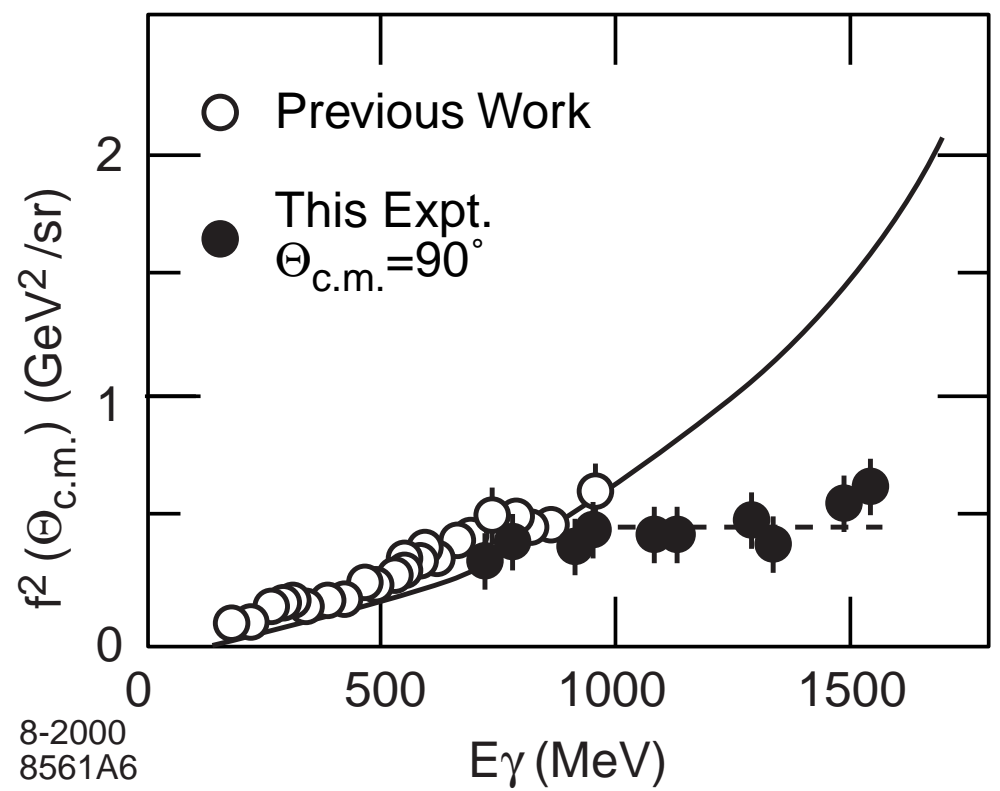

Figure 7: Comparison of deuteron photodisintegration data with the scaling prediction which requires $f^{2}\left(\theta_{c m}\right)$ to be at most logarithmically dependent on energy at large momentum transfer. The data in are from Belz et al. [85] The solid curve is a nuclear physics prediction 86$]$. 
The postulate that the QCD coupling has an infrared fixed-point provides an understanding of the applicability of conformal scaling and constituent counting rules to physical QCD processes 12, 13. The general success of dimensional counting rules implies that the effective coupling $\alpha_{V}\left(Q^{*}\right)$ controlling the gluon exchange propagators in $T_{H}$ are frozen in the infrared, since the effective momentum transfers $Q^{*}$ exchanged by the gluons are often a small fraction of the overall momentum transfer [34]. In this case, the pinch contributions are suppressed by a factor decreasing faster than a fixed power [12]. The effective coupling $\alpha_{\tau}(s)$ extracted from $\tau$ decays displays a flat behavior at low mass scales 87.

The field of analyzable exclusive processes has been expanded to a wide range of QCD processes, such as electroweak decay amplitudes, highly virtual diffractive processes such as $\gamma^{*} p \rightarrow \rho p$ 88, 89, and semi-exclusive processes such as $\gamma^{*} p \rightarrow \pi^{+} X$ [101 where the $\pi^{+}$is produced in isolation at large $p_{T}$. An important new application is the recent analysis of hard exclusive $B$ decays by Beneke et al. [2] and Keum et al. [3] Deeply virtual Compton amplitude $\gamma^{*} p \rightarrow \gamma p$ has emerged as one of the most important exclusive QCD reactions 90, 91, 92, 93]. The process factorizes into a hard amplitude representing Compton scattering on the quark times skewed parton distributions. The resulting skewed parton form factors can be represented as diagonal and off-diagonal convolutions of light-cone wavefunctions, as in semileptonic $B$ decay [94]. New sum rules can be constructed which correspond to gravitons coupling to the quarks of the proton 90. It is possible that the handbag approximation to DVCS may be modified by corrections to the quark propagator similar to those which appear in the final state interaction corrections to deep inelastic scattering 95, 96. In particular, one can expect that the propagator corrections will give single-spin asymmetries correlating the spin of the proton with the normal to the production plane in DVCS [97.

The hard diffraction of vector mesons $\gamma^{*} p \rightarrow V^{0} p$ at high $Q^{2}$ and high energies for longitudinally polarized vector mesons factorizes into a skewed parton distribution times the hard scale $\gamma^{*} g \rightarrow g V^{0}$ amplitude, where the physics of the vector meson is contained in its distribution amplitude [88, 21, 98]. The data appears consistent with the $s, t$ and $Q^{2}$ dependence predicted by theory. Ratios of these processes for different mesons are sensitive to the ratio of $1 / x$ moments of the $V^{0}$ distribution amplitudes.

The two-photon annihilation process $\gamma^{*} \gamma \rightarrow$ hadrons, which is measurable in single-tagged $e^{+} e^{-} \rightarrow e^{+} e^{-}$hadrons events provides a semi-local probe of even charge conjugation $C={ }^{+}$hadron systems $\pi^{0}, \eta^{0}, \eta^{\prime}, \eta_{c}, \pi^{+} \pi^{-}$, etc. The $\gamma^{*} \gamma \rightarrow \pi^{+} \pi^{-}$hadron pair process is related to virtual Compton scattering on a pion target by crossing. Hadron pair production is of particular interest since the leading-twist amplitude is sensitive to the $1 / x-1 /(1-x)$ moment of the two-pion distribution amplitude coupled to two valence quarks [21, 99]. This type of measurement can also constrain the parameters of the effective chiral theory, which is needed for example to constrain the hadronic light-by-light contribution to the muon magnetic moment 100.

One can also study hard "semi-exclusive" processes [101] of the form $A+B \rightarrow C+Y$ 
which are characterized by a large momentum transfer between the particles $A$ and $C$ and a large rapidity gap between the final state particle $C$ and the inclusive system $Y$. Such reactions are in effect generalizations of deep inelastic lepton scattering, providing novel currents which probe specific quark distributions of the target $B$ at fixed momentum fraction and novel spin-dependent parton distributions.

\section{Exact Formulae for Exclusive Processes}

The natural formalism for describing the hadronic wavefunctions which enter exclusive and diffractive amplitudes is the light-cone Fock representation obtained by quantizing the theory at fixed "light-cone" time $\tau=t+z / c$ [102]. For example, the proton state has the Fock expansion

$$
\begin{aligned}
|p\rangle= & \sum_{n}\langle n \mid p\rangle|n\rangle \\
= & \psi_{3 q / p}^{(\Lambda)}\left(x_{i}, \vec{k}_{\perp i}, \lambda_{i}\right)|u u d\rangle \\
& +\psi_{3 q g / p}^{(\Lambda)}\left(x_{i}, \vec{k}_{\perp i}, \lambda_{i}\right)|u u d g\rangle+\cdots
\end{aligned}
$$

representing the expansion of the exact QCD eigenstate on a non-interacting quark and gluon basis. The probability amplitude for each such $n$-particle state of on-mass shell quarks and gluons in a hadron is given by a light-cone Fock state wavefunction $\psi_{n / H}\left(x_{i}, \vec{k}_{\perp i}, \lambda_{i}\right)$, where the constituents have longitudinal light-cone momentum fractions $x_{i}=k_{i}^{+} / p^{+}=\left(k_{i}^{0}+k_{i}^{z}\right) /\left(p^{0}+p^{z}\right), \sum_{i=1}^{n} x_{i}=1$, relative transverse momentum $\vec{k}_{\perp i}, \sum_{i=1}^{n} \vec{k}_{\perp i}=\overrightarrow{0}_{\perp}$, and helicities $\lambda_{i}$. The effective lifetime of each $n$ - parton configuration in the laboratory frame is $\frac{2 P_{l a b}}{\mathcal{M}_{n}^{2}-M_{p}^{2}}$ where $\mathcal{M}_{n}^{2}=\sum_{i=1}^{n}\left(k_{\perp i}^{2}+m_{i}^{2}\right) / x_{i}<\Lambda^{2}$ is the off-shell invariant mass and $\Lambda$ is a global ultraviolet regulator. A crucial feature of the light-cone formalism is the fact that the form of the $\psi_{n / H}^{(\Lambda)}\left(x_{i}, \vec{k}_{\perp i}, \lambda_{i}\right)$ is invariant under longitudinal boosts; i.e., the light-cone wavefunctions expressed in the relative coordinates $x_{i}$ and $k_{\perp i}$ are independent of the total momentum $P^{+}, \vec{P}_{\perp}$ of the hadron. Angular momentum conservation also has a precise meaning in the light-front representation: for each $n$-particle Fock state,

$$
J^{z}=\sum_{i=1}^{n} S_{i}^{z}+\sum_{i}^{n-1} L_{i}^{z}
$$

since there are only $n-1$ relative orbital angular momenta 96.

Matrix elements of space-like local operators for the coupling of photons, gravitons and the deep inelastic structure functions can all be expressed as overlaps of light-cone wavefunctions with the same number of Fock constituents. This is possible since one can choose the special frame $q^{+}=0$ [27, 28] for space-like momentum transfer and take matrix elements of "plus" components of currents such as $J^{+}$and $T^{++}$. Since the physical vacuum in light-cone quantization coincides with the perturbative vacuum, 
no contributions to matrix elements from vacuum fluctuations occur 102. In the lightcone formalism one can identify the Dirac and Pauli form factors from the light-cone spin-conserving and spin-flip vector current matrix elements of the $J^{+}$current [29]: $\left\langle P+q, \uparrow\left|\frac{J^{+}(0)}{2 P^{+}}\right| P, \uparrow\right\rangle=F_{1}\left(q^{2}\right),\left\langle P+q, \uparrow\left|\frac{J^{+}(0)}{2 P^{+}}\right| P, \downarrow\right\rangle=-\frac{\left(q^{1}-\mathrm{i} q^{2}\right)}{2 M} F_{2}\left(q^{2}\right)$. More explicitly, the Pauli form factor can be calculated from the expression

$$
-\left(q^{1}-\mathrm{i} q^{2}\right) \frac{F_{2}\left(q^{2}\right)}{2 M}=\sum_{a} \int \frac{\mathrm{d}^{2} \vec{k}_{\perp} \mathrm{d} x}{16 \pi^{3}} \sum_{j} e_{j} \psi_{a}^{\uparrow *}\left(x_{i}, \vec{k}_{\perp i}^{\prime}, \lambda_{i}\right) \psi_{a}^{\downarrow}\left(x_{i}, \vec{k}_{\perp i}, \lambda_{i}\right),
$$

where the summation is over all contributing Fock states $a$ and struck constituent charges $e_{j}$. The arguments of the final-state light-cone wavefunction are [27, 28] $\vec{k}_{\perp i}^{\prime}=$ $\vec{k}_{\perp i}+\left(1-x_{i}\right) \vec{q}_{\perp}$ for the struck constituent and $\vec{k}_{\perp i}^{\prime}=\vec{k}_{\perp i}-x_{i} \vec{q}_{\perp}$ for each spectator. The Pauli form factor couples Fock states differing by one unit of orbital angular momentum, since the initial and final states have opposite total spin $J_{z}$ and the constituent spins are unchanged in the overlap formula. In effect, one is measuring the spin-orbit $\vec{S} \cdot \vec{L}$ in the light-front formalism. Thus the $F_{2}$ form factor and the anomalous moment directly measure relative angular momentum in the proton. This is also true for the $E$ generalized parton distribution determined in DVCS and the single-spin asymmetries measured in DIS. In these cases, the quark contributions are weighted by the square of the quark charges.

In the ultra-relativistic limit where the radius of the system is small compared to its Compton scale $1 / M$, the anomalous magnetic moment must vanish 103. The light-cone formalism is consistent with this theorem. The anomalous moment coupling $B(0)$ to a graviton vanishes for any composite system. This remarkable result, first derived by Okun and Kobzarev 104, 105, 106, 107, follows directly from the Lorentz boost properties of the light-cone Fock representation [96].

The overlap formula for the form factors is invariant under $\vec{q}_{\perp} \rightarrow-\vec{q}_{\perp}$. Thus at large momentum transfer one obtains an expansion of form factors in powers of $1 / q^{2}$ modulo logarithms. This also can be seen from a twist expansion of the operator product expansion[108].

Exclusive semi-leptonic $B$-decay amplitudes involving time-like currents such as $B \rightarrow A \ell \bar{\nu}$ can also be evaluated exactly in the light-cone framework [109, [10]. In this case, the $q^{+}=0$ frame cannot be used, and the time-like decay matrix elements require the computation of both the diagonal matrix element $n \rightarrow n$ where parton number is conserved and the off-diagonal $n+1 \rightarrow n-1$ convolution such that the current operator annihilates a $q \overline{q^{\prime}}$ pair in the initial $B$ wavefunction. See Fig. 2(h). A similar result holds for the light-cone wavefunction representation of the deeply virtual Compton amplitude [94]. This feature will carry over to exclusive hadronic $B$-decays, such as $B^{0} \rightarrow \pi^{-} D^{+}$. In this case the pion can be produced from the coalescence of a $d \bar{u}$ pair emerging from the initial higher particle number Fock wavefunction of the $B$. The $D$ meson is then formed from the remaining quarks after the internal exchange of a $W$ boson. 
In principle, a precise evaluation of the hadronic matrix elements needed for $B$ decays and other exclusive electroweak decay amplitudes requires knowledge of all of the light-cone Fock wavefunctions of the initial and final state hadrons. In the case of model gauge theories such as QCD $(1+1)$ [111 or collinear QCD 112 in one-space and one-time dimensions, the complete evaluation of the light-cone wavefunction is possible for each baryon or meson bound-state using the DLCQ method[113, 112]. It would be interesting to use such solutions as a model for physical $B$-decays.

There are now real prospects of computing the hadron wavefunctions and distribution amplitudes from first principles in QCD as exemplified by the computation 114 of the pion distribution amplitude using a combination of DLCQ and the transverse lattice methods and recent results from traditional lattice gauge theory [115]. Instanton models predict a pion distribution amplitude close to the asymptotic form 116. A new result for the proton distribution amplitude treating nucleons as chiral solitons has recently been derived by Diakonov and Petrov [117. Dyson-Schwinger models 118 of hadronic Bethe-Salpeter wavefunctions can also be used to predict light-cone wavefunctions and hadron distribution amplitudes by integrating over the relative $k^{-}$momentum.

\section{Color Transparency}

Each hadron entering or emitted from a hard exclusive reaction initially emerges with high momentum and small transverse size $b_{\perp}=\mathcal{O}(1 / \widetilde{Q})$. A fundamental feature of gauge theory is that soft gluons decouple from the small color-dipole moment of the compact fast-moving color-singlet wavefunction configurations of the incident and final-state hadrons. The transversely compact color-singlet configurations can effectively persist over a distance of order $\ell_{\text {Ioffe }}=\mathcal{O}\left(E_{\text {lab }} / Q^{2}\right)$, the Ioffe coherence length. Thus if we study hard quasi-elastic processes in a nuclear target such as $e A \rightarrow e^{\prime} p^{\prime}(A-1)$ or $p A \rightarrow p^{\prime}(A-1)$, the outgoing and ingoing hadrons will have minimal absorption in a nucleus. The diminished absorption of hadrons produced in hard exclusive reactions implies additivity of the nuclear cross section in nucleon number $A$ and is the theoretical basis for the "color transparency" of hard quasi-elastic reactions [24, 119, 120, 121]. In contrast, in conventional Glauber scattering, one predicts strong, nearly energy-independent initial and final state attenuation. Similarly, in hard diffractive processes such as $\gamma^{*}\left(Q^{2}\right) p \rightarrow \rho p$ [88] only the small transverse configurations $b_{\perp} \sim 1 / Q$ of the longitudinally polarized vector meson distribution amplitude is involved. Its hadronic interactions as it exits the nucleus will be minimal, and thus the $\gamma^{*}\left(Q^{2}\right) N \rightarrow \rho N$ reaction can occur coherently throughout a nuclear target in reactions without absorption or shadowing. Evidence for color transparency in such reactions has been reported by Fermilab experiment E665 122.

The most convincing demonstration of color transparency has been reported by the E791 group at FermiLab [123] in measurements of diffractive dissociation of a high energy pions to high transverse momentum dijets; $\pi A \rightarrow$ jet jet $A$; the forward 
diffractive amplitude is observed to grow in proportion to the total number of nucleons in the nucleus, in strong contrast to standard Glauber theory which predicts that only the front surface of the nucleus should be effective.

There is also evidence for the onset of color transparency in large angle quasielastic $p p$ scattering in nuclear targets 124, 125, 126], in the regime $6<s<25 \mathrm{GeV}^{2}$, indicating that small wavefunction configurations are indeed controlling this exclusive reaction at moderate momentum transfers. However at $p_{\text {lab }} \simeq 12 \mathrm{GeV}, E_{c m} \simeq 5 \mathrm{GeV}$, color transparency dramatically fails. It is noteworthy that in the same energy range, the normal-normal spin asymmetry $A_{N N}$ in elastic $p p \rightarrow p p$ scattering at $\theta_{c m}=90^{\circ}$ increases dramatically to $A_{N N} \simeq 0.6$ - it is about four times more probable that the protons scatter with helicity normal to the scattering plane than anti-normal 127.

The unusual spin and color transparency effects seen in elastic proton-proton scattering at $E_{C M} \sim 5 \mathrm{GeV}$ and large angles could be related to the charm threshold and the effects of a $|u u d u u d c \bar{c}\rangle$ resonance which would appear as in the $J=L=S=1$ $p p$ partial wave 128, 129]. The intermediate state $|u u d u u d c \bar{c}\rangle$ has odd intrinsic parity and couples to the $J=S=1$ initial state, thus strongly enhancing scattering when the incident projectile and target protons have their spins parallel and normal to the scattering plane. A similar enhancement of $A_{N N}$ is observed at the strangeness threshold. The physical protons coupling at the charm threshold will have normal Glauber interactions, thus explaining the anomalous change in color transparency observed at the same energy in quasi-elastic $p p$ scattering. A crucial test of the charm hypothesis is the observation of open charm production near threshold with a cross section of order of $1 \mu \mathrm{b}$ [128, 129]. A similar cross section is expected for the second threshold for open charm production from $p \bar{p} \rightarrow$ charm $p \bar{p}$. An alternative explanation of the color transparency and spin anomalies in $p p$ elastic scattering has been postulated by Ralston, Jain, and Pire 130, 120. The oscillatory effects in the large-angle $p p \rightarrow p p$ cross section and spin structure are postulated to be due to the interference of Landshoff pinch and perturbative QCD amplitudes. In the case of quasi-elastic reactions, the nuclear medium absorbs and filters out the non-compact pinch contributions, leaving the additive hard contributions unabsorbed. It is clearly important that these two alternative explanations be checked by experiment.

In general, one can expect strong effects whenever heavy quarks are produced at low relative velocity with respect to each other or the other quarks in the reaction since the QCD van der Waals interactions become maximal in this domain. The opening of the strangeness and charm threshold in intermediate states can become most apparent in large angle reactions such as $p p$ scattering and pion photoproduction since the competing perturbative QCD amplitudes are power-suppressed. Charm and bottom production near threshold such as $J / \psi$ photoproduction is also sensitive to the multiquark, gluonic, and hidden-color correlations of hadronic and nuclear wavefunctions in QCD since all of the target's constituents must act coherently within the small interaction volume of the heavy quark production subprocess 131. Although such multi-parton subprocess cross sections are suppressed by powers of $1 / m_{Q}^{2}$, they have 
less phase-space suppression and can dominate the contributions of the leading-twist single-gluon subprocesses in the threshold regime.

\section{Self-Resolved Diffractive Reactions and Light Cone Wavefunctions}

Diffractive multi-jet production in heavy nuclei provides a novel way to measure the shape of the LC Fock state wavefunctions and test color transparency. For example, consider the reaction[132, 133, 134] $\pi A \rightarrow \mathrm{Jet}_{1}+\mathrm{Jet}_{2}+A^{\prime}$ at high energy where the nucleus $A^{\prime}$ is left intact in its ground state. The transverse momenta of the jets balance so that $\vec{k}_{\perp i}+\vec{k}_{\perp 2}=\vec{q}_{\perp}<R_{A}^{-1}$. The light-cone longitudinal momentum fractions also need to add to $x_{1}+x_{2} \sim 1$ so that $\Delta p_{L}<R_{A}^{-1}$. The process can then occur coherently in the nucleus. Because of color transparency, the valence wavefunction of the pion with small impact separation, will penetrate the nucleus with minimal interactions, diffracting into jet pairs 132. The $x_{1}=x, x_{2}=1-x$ dependence of the di-jet distributions will thus reflect the shape of the pion valence light-cone wavefunction in $x$; similarly, the $\vec{k}_{\perp 1}-\vec{k}_{\perp 2}$ relative transverse momenta of the jets gives key information on the derivative of the underlying shape of the valence pion wavefunction 133, 134, 135, 136. The diffractive nuclear amplitude extrapolated to $t=0$ should be linear in nuclear number $A$ if color transparency is correct. The integrated diffractive rate should then scale as $A^{2} / R_{A}^{2} \sim A^{4 / 3}$ as verified by E791 for $500 \mathrm{GeV}$ incident pions on nuclear targets 123. The measured momentum fraction distribution of the jets [137] is consistent with the shape of the pion asymptotic distribution amplitude, $\phi_{\pi}^{\text {asympt }}(x)=\sqrt{3} f_{\pi} x(1-x)$. Data from CLEO 138 for the $\gamma \gamma^{*} \rightarrow \pi^{0}$ transition form factor also favor a form for the pion distribution amplitude close to the asymptotic solution[17, 11] to the perturbative QCD evolution equation.

The diffractive dissociation of a hadron or nucleus can also occur via the Coulomb dissociation of a beam particle on an electron beam (e.g. at HERA or eRHIC) or on the strong Coulomb field of a heavy nucleus (e.g. at RHIC or nuclear collisions at the LHC) 135. The amplitude for Coulomb exchange at small momentum transfer is

proportional to the first derivative $\sum_{i} e_{i} \frac{\partial}{\vec{k}_{T i}} \psi$ of the light-cone wavefunction, summed over the charged constituents. The Coulomb exchange reactions fall off less fast at high transverse momentum compared to pomeron exchange reactions since the lightcone wavefunction is effective differentiated twice in two-gluon exchange reactions. It is also interesting to study diffractive tri-jet production using proton beams $p A \rightarrow$ $\mathrm{Jet}_{1}+\mathrm{Jet}_{2}+\mathrm{Jet}_{3}+A^{\prime}$ to determine the fundamental shape of the 3-quark structure of the valence light-cone wavefunction of the nucleon at small transverse separation 133.

There has been an important debate whether diffractive jet production faithfully measures the light-front wavefunctions of the projectile. Braun et al. 139 and Chernyak [140] have argued that one should systematically iterate the gluon exchange kernel from all sources, including final state interactions. Thus if the hard momen- 
tum exchange which produces the high transverse momentum di-jets occurs in the final state, then the $x$ and $k_{\perp}$ distributions will reflect the gluon exchange kernel, not the pion's wavefunction. However, it should be noted that the measurements of pion diffraction by the E791 experiment 137 are performed on a platinum target. Only the part of the pion wavefunction with small impact separation can give the observed color transparency; i.e., additivity of the amplitude on nuclear number. Thus the nucleus automatically selects events where the jets are produced at high transverse momentum in the initial state before the pion reaches the nucleus 132.

The debate[141, 139, 140] concerning the nature of diffractive dijet dissociation also applies to the simpler analysis of diffractive dissociation via Coulomb exchange. The one-photon exchange matrix element can be identified with the spacelike electromagnetic form factor for $\pi \rightarrow q \bar{q} ;\left\langle\pi ; P-q\left|j^{+}(0)\right| q \bar{q} ; P\right\rangle$. Here the state $|q \bar{q}\rangle$ is the eigenstate of the QCD Hamiltonian; it is effectively an 'out' state. If we choose the $q^{+}=0$ frame where $q^{2}=-\vec{q}_{\perp}^{2}$, then the form factor is exactly the overlap integral in transverse momentum of the pion and $\bar{q} q$ LCWFs summed over Fock States. The form factor vanishes at $Q^{2}=0$ because it is the matrix element of the total charge operator and the pion and jet-jet eigenstates are orthogonal. The $n=2$ contribution to the form factor is the convolution $\psi_{\pi}\left(x, k_{\perp}-(1-x) q_{\perp}\right)$ with $\psi_{\bar{q} q}\left(x, k_{\perp}\right)$. This can be expanded at small $q^{2}$ in terms of the transverse momentum derivatives of the pion wavefunction. The final-state wavefunction represents an outgoing wave of free quarks with momentum $y, \ell_{\perp}$ and $1-y,-\ell_{\perp}$. To first approximation the wavefunction $\psi_{\bar{q} q}\left(x, k_{\perp}\right)$ peaks strongly at $x=y$ and $k_{\perp}=\ell_{\perp}$. Using this approximation, the form factor at small $Q^{2}$ is proportional to the derivative of the pion light-cone wavefunction $\left[e_{q}(1-x)-e_{\bar{q}} x\right] \frac{\partial}{d k_{\perp}} \psi_{\pi}\left(x, k_{\perp}\right)$ evaluated at $x=y$ and $k_{\perp}=\ell_{\perp}$. One can also consider corrections to the final state wavefunction from gluon exchange. However, the final quarks are already moving in the correct direction at zeroth order, so these corrections would be expected to be of higher order.

\section{Conclusions}

Perturbative QCD provides an important guide to high momentum transfer exclusive processes. The theory involves fundamental details of hadron structure at the amplitude level. The hadron wavefunctions required for these perturbative QCD analyses are also relevant for computing exclusive heavy hadron decays.

The leading-twist contributions to exclusive amplitudes derive from the kinematic regime where the quarks and gluons propagators are evaluated in the perturbative regime. There are many successes of the perturbative approach, including important checks of color transparency and hadron helicity conservation. The successes of perturbative QCD scaling for exclusive processes at presently accessible momentum transfers can be understood if the effective QCD coupling is approximately constant at the momentum transfers scales relevant to the hard scattering amplitudes. The Sudakov suppression of the long-distance contributions is strengthened if the coupling 
is frozen because it involves the exponentiation of a double logarithmic series.

In this review I have argued that the new Jefferson Laboratory measurements of the ratio of proton form factors are not necessarily incompatible with the perturbative QCD predictions. I have also argued that the apparent discrepancy of theory with the normalization of the spacelike pion form factor may be due to the difficulty of extrapolating electroproduction data from the off-shell regime to the pion pole.

Further experimental studies, particularly measurements of electroproduction at Jefferson Laboratory and the study of two-photon exclusive channels at CLEO and the B-factories have the potential of providing critical information on the hadron wavefunctions as well as testing the dominant dynamical processes at short distances. Testing quantum chromodynamics to high precision in exclusive processes is not easy. Virtually all QCD processes are complicated by the presence of dynamical higher twist effects, including power-law suppressed contributions due to multi-parton correlations, intrinsic transverse momentum, and finite quark masses. Many of these effects are inherently nonperturbative in nature and require detailed knowledge of hadron wavefunctions themselves. New systematic approaches to higher twist contributions are required, such as the recent development of effective field theories [142, 143].

Diffractive dijet production on nuclei has provided a compelling demonstration of color transparency and because of the color filtering effect of the nuclear target has yielded strong empirical constraints on the shape of pion distribution amplitude. I have argued that these "self-resolving" diffractive processes can also provide direct experimental information on the light-cone wavefunctions of the photon and proton in terms of their QCD degrees of freedom, as well as the composition of nuclei in terms of their nucleon and mesonic degrees of freedom.

\section{Acknowledgements}

I am grateful to Anatoly Radyushkin and Paul Stoler for their kind invitation to this very interesting and provocative workshop. I also thank Volodya Braun, Carl Carlson, Markus Diehl, Lyonya Frankfurt, Haiyan Gao, Susan Gardner, Gudrun Hiller, John Hiller, Paul Hoyer, Dae Sung Hwang, Peter Kroll, Jerry Miller, Kolya Nilolaev, Stephane Peigne, Mark Strikman, and Christian Weiss for helpful conversations.

\section{References}

[1] For reviews, see S. J. Brodsky and G. P. Lepage, SLAC-PUB-4947 In *A.H. Mueller, (ed): Perturbative Quantum Chromodynamics, 1989, p. 93-240, and S. J. Brodsky, SLAC-PUB-8649 In *Shifman, M. (ed.): At the frontier of particle physics, vol. 2* 1343-1444. 
[2] M. Beneke, G. Buchalla, M. Neubert and C. T. Sachrajda, Nucl. Phys. B 591, 313 (2000) arXiv:hep-ph/0006124.

[3] Y. Y. Keum, H. N. Li and A. I. Sanda, Phys. Rev. D 63, 054008 (2001) arXiv:hep-ph/0004173. 0004173;

[4] A. Szczepaniak, E. M. Henley and S. J. Brodsky, Phys. Lett. B 243, 287 (1990).

[5] A review of QCD analyses of exclusive $B$ decays is given in S. J. Brodsky, arXiv:hep-ph/0104153].

[6] C. K. Chua, W. S. Hou and S. Y. Tsai, arXiv:hep-ph/0204185.

[7] For a review of QCD tests in photon-photon collisions see, S. J. Brodsky, in Proc. of the $e^{+} e^{-}$Physics at Intermediate Energies Conference ed. Diego Bettoni, eConf C010430, W01 (2001) arXiv:hep-ph/0106294.

[8] M. K. Jones [Jefferson Lab Hall A Collaboration], Nucl. Phys. A 699, 124 (2002). O. Gayou et al. [Jefferson Lab Hall A Collaboration], Phys. Rev. Lett. 88, 092301 (2002) arXiv:nucl-ex/0111010. Phys. Rev. Lett. 84, 1398 (2000) arXiv:nuclex/9910005].

[9] S. J. Brodsky and G. P. Lepage, Phys. Rev. D24, 2848 (1981).

[10] V. Chernyak, arXiv:hep-ph/9906387.

[11] G. P. Lepage and S. J. Brodsky, Phys. Rev. D 22, 2157 (1980).

[12] S. J. Brodsky and G. R. Farrar, Phys. Rev. D 11, 1309 (1975).

[13] V. A. Matveev, R. M. Muradian and A. N. Tavkhelidze, Lett. Nuovo Cim. 7, 719 (1973).

[14] P. V. Landshoff, Phys. Rev. D 10, 1024 (1974).

[15] S. J. Brodsky and G. P. Lepage, SLAC-PUB-2294 Workshop on Current Topics in High Energy Physics, Cal Tech., Pasadena, Calif., Feb 13-17, 1979.

[16] G. P. Lepage and S. J. Brodsky, Momentum Transfer," Phys. Rev. Lett. 43, 545 (1979).

[17] G. P. Lepage and S. J. Brodsky, Phys. Lett. B 87, 359 (1979).

[18] A. V. Efremov and A. V. Radyushkin, Theor. Math. Phys. 42 (1980) 97;

[19] J. Polchinski and M. J. Strassler, Phys. Rev. Lett. 88, 031601 (2002) arXiv:hepth/0109174. 
[20] S. J. Brodsky, Y. Frishman, G. P. Lepage and C. Sachrajda, Phys. Lett. 91B, 239 (1980).

[21] D. Muller, Phys. Rev. D 51, 3855 (1995) [arXiv:hep-ph/9411338].

[22] P. Ball and V. M. Braun, Nucl. Phys. B 543, 201 (1999) arXiv:hep-ph/9810475.

[23] V. M. Braun, S. E. Derkachov, G. P. Korchemsky and A. N. Manashov, Nucl. Phys. B553, 355 (1999) arXiv:hep-ph/9902375.

[24] S. J. Brodsky and A. H. Mueller, Phys. Lett. B206, 685 (1988).

[25] S. J. Brodsky, C. R. Ji and G. P. Lepage, Phys. Rev. Lett. 51, 83 (1983).

[26] S. J. Brodsk y and H. J. Lu, Phys. Rev. D 51, 3652 (1995) arXiv:hep$\mathrm{ph} / 9405218]$.

[27] S. D. Drell and T. Yan, Phys. Rev. Lett. 24, 181 (1970).

[28] G. B. West, Phys. Rev. Lett. 24, 1206 (1970).

[29] S. J. Brodsky and S. D. Drell, Phys. Rev. D 22, 2236 (1980).

[30] V. L. Chernyak, A. R. Zhitnitsky and V. G. Serbo, JETP Lett. 26, 594 (1977).

[31] V. L. Chernyak, V. G. Serbo and A. R. Zhitnitsky, Sov. J. Nucl. Phys. 31, 552 (1980).

[32] G. R. Farrar and D. R. Jackson, Phys. Rev. Lett. 43, 246 (1979).

[33] A. Duncan and A. H. Mueller, Phys. Rev. D21, 1636 (1980).

[34] S. J. Brodsky, C. R. Ji, A. Pang and D. G. Robertson, Phys. Rev. D 57, 245 (1998) arXiv:hep-ph/9705221.

[35] M. Beneke, arXiv:hep-ph/0202056.

[36] D. Muller, D. Robaschik, B. Geyer, F. M. Dittes and J. Horejsi, Fortsch. Phys. 42, 101 (1994) arXiv:hep-ph/9812448.

[37] B. Melic, B. Nizic and K. Passek, Phys. Rev. D 60, 074004 (1999) arXiv:hep$\mathrm{ph} / 9802204$.

[38] A. Szczepaniak, A. Radyushkin and C. Ji, Phys. Rev. D57, 2813 (1998) arXiv:hep-ph/9708237.

[39] A. S. Kronfeld and B. Nizic, Phys. Rev. D44, 3445 (1991). 
[40] P. A. Guichon and M. Vanderhaeghen, Prog. Part. Nucl. Phys. 41, 125 (1998) arXiv:hep-ph/9806305.

[41] T. C. Brooks and L. J. Dixon, Phys. Rev. D 62, 114021 (2000) arXiv:hep$\mathrm{ph} / 0004143$.

[42] M. A. Shupe et al., Phys. Rev. D 19, 1921 (1979).

[43] N. Isgur and C. H. Llewellyn Smith, Phys. Lett. B217, 535 (1989).

[44] A. V. Radyushkin, Phys. Rev. D58, 114008 (1998) hep-ph/9803316.

[45] J. Bolz and P. Kroll, Z. Phys. A356, 327 (1996) hep-ph/9603289.

[46] M. Diehl, T. Feldmann, R. Jakob and P. Kroll, Eur. Phys. J. C 8, 409 (1999) arXiv:hep-ph/9811253.

[47] H. W. Huang, P. Kroll and T. Morii, Eur. Phys. J. C 23, 301 (2002) arXiv:hepph/0110208.

[48] G. P. Lepage, S. J. Brodsky, T. Huang and P. B. Mackenzie, and S. J. Brodsky, T. Huang and G. P. Lepage, In *Banff 1981, Proceedings, Particles and Fields 2*, 143-199.

[49] H. N. Li and G. Sterman, Nucl. Phys. B 381, 129 (1992).

[50] S. J. Brodsky, F. E. Close and J. F. Gunion, Phys. Rev. D6, 177 (1972).

[51] C. Weiss, arXiv:hep-ph/0206295.

[52] M. E. Peskin, Phys. Lett. B88, 128 (1979).

[53] C-R Ji, A. F. Sill and R. M. Lombard-Nelsen, Phys. Rev. D36, 165 (1987).

[54] V. L. Chernyak and I. R. Zhitnitsky, Nucl. Phys. B246, 52 (1984).

[55] V. L. Chernyak, A. A. Ogloblin and I. R. Zhitnitsky, Z. Phys. C42, 583 (1989).

[56] I. D. King and C. T. Sachrajda, Nucl. Phys. B279, 785 (1987).

[57] M. Gari and N. Stefanis, Phys. Lett. B175, 462 (1986), M. Gari and N. Stefanis, Phys. Lett. 187B, 401 (1987).

[58] N. G. Stefanis, Eur. Phys. J. C7, 1 (1999) arXiv:hep-ph/9911375.

[59] S. J. Brodsky, J. R. Ellis, J. S. Hagelin and C. T. Sachrajda, Nucl. Phys. B 238, 561 (1984).

[60] Y. Kuramashi [JLQCD Collaboration], arXiv:hep-ph/0103264]. 
[61] S. J. Brodsky, G. P. Lepage and S. A. Zaidi, Phys. Rev. D23, 1152 (1981).

[62] C. E. Carlson, Phys. Rev. D34, 2704 (1986).

[63] P. Stoler, Phys. Rept. 226, 103 (1993).

[64] P. V. Pobylitsa, V. Polyakov and M. Strikman, Phys. Rev. Lett. 87, 022001 (2001) arXiv:hep-ph/0101279.

[65] M. Ambrogiani et al. [E835 Collaboration], Phys. Rev. D 60, 032002 (1999).

[66] S. Brodsky, D. S. Hwang, and J. Hiller, in preparation.

[67] S. J. Brodsky and M. Karliner, Phys. Rev. Lett. 78, 4682 (1997), hepph/9704379.

[68] S. J. Brodsky and S. Gardner, Phys. Rev. D 65, 054016 (2002) arXiv:hepph/0108121.

[69] C. H. Chang and W. S. Hou, Phys. Rev. D 64, 071501 (2001).

[70] R. L. Anderson et al., Phys. Rev. Lett. 30, 627 (1973)

[71] H. J. Besch, F. Krautschneider, K. P. Sternemann and W. Vollrath, Z. Phys. C 16, 1 (1982).

[72] W. Melnitchouk, Nucl. Phys. A 699, 278 (2002) arXiv:hep-ph/0106262.

[73] M. Diehl, P. Kroll and C. Vogt, Phys. Lett. B 532, 99 (2002) arXiv:hepph/0112274.

[74] J. Boyer et al., Phys. Rev. Lett. 56, 207 (1980); TPC/Two Gamma Collaboration (H. Aihara et al.), Phys. Rev. Lett. 57, 404 (1986).

[75] S. J. Brodsky and G. P. Lepage, Phys. Rev. D24, 1808 (1981).

[76] H. Paar et al., CLEO collaboration (to be published). V. Savinov, in Proc. of the $e^{+} e^{-}$Physics at Intermediate Energies Conference ed. Diego Bettoni, eConf C010430, W03 (2001) arXiv:hep-ex/0106013. See also D. Morgan, M. R. Pennington and M. R. Whalley, DPDG-94-01. H. Aihara et al. [TPC/Two Gamma Collaboration], Phys. Rev. D 40 (1989) 2772. K. Grzelak [DELPHI Collaboration], Nucl. Phys. Proc. Suppl. 82, 316 (2000).

[77] J. F. Gunion, S. J. Brodsky and R. Blankenbecler, Phys. Rev. D8, 287 (1973).

[78] R. Blankenbecler, S.J. Brodsky, J. F. Gunion and R. Savit, Phys. Rev. D8, 4117 (1973). 
[79] D. Sivers, S. J. Brodsky and R. Blankenbecler, Phys. Rept. 23, 1 (1976).

[80] S. J. Brodsky and B. T. Chertok, Phys. Rev. D14, 3003 (1976).

[81] G. R. Farrar, K. Huleihel and H. Zhang, Phys. Rev. Lett. 74, 650 (1995).

[82] R. J. Holt, Phys. Rev. C41, 2400 (1990).

[83] C. Bochna et al. [E89-012 Collaboration], Phys. Rev. Lett. 81, 4576 (1998) arXiv:nucl-ex/9808001.

[84] S. J. Brodsky and J. R. Hiller, Phys. Rev. C28, 475 (1983).

[85] J. E. Belz et al., Phys. Rev. Lett. 74, 646 (1995).

[86] T. S. Lee, CONF-8805140-10.

[87] S. J. Brodsky, S. Menke, and J. Rathman (in preparation).

[88] S. J. Brodsky, L. Frankfurt, J. F. Gunion, A. H. Mueller and M. Strikman, Phys. Rev. D50, 3134 (1994), arXiv:hep-ph/9402283.

[89] J. C. Collins, Phys. Rev. D 57, 3051 (1998) [Erratum-ibid. D 61, 019902 (2000)] arXiv:hep-ph/9709499.

[90] X. Ji, Phys. Rev. D55, 7114 (1997), arXiv:hep-ph/9609381.

[91] A. V. Radyushkin, Phys. Rev. D56, 5524 (1997) hep-ph/9704207.

[92] M. Diehl, T. Feldmann, R. Jakob and P. Kroll, Phys. Lett. B460, 204 (1999) arXiv:hep-ph/9903268.

[93] M. Diehl, T. Feldmann, R. Jakob and P. Kroll, Eur. Phys. J. C8, 409 (1999), arXiv:hep-ph/9811253.

[94] S. J. Brodsky, M. Diehl and D. S. Hwang, Nucl. Phys. B 596, 99 (2001) arXiv:hep-ph/0009254.

[95] S. J. Brodsky, P. Hoyer, N. Marchal, S. Peigne and F. Sannino, Phys. Rev. D 65, 114025 (2002) arXiv:hep-ph/0104291.

[96] S. J. Brodsky, D. S. Hwang, B. Q. Ma and I. Schmidt, Nucl. Phys. B 593, 311 (2001) arXiv:hep-th/0003082.

[97] S. J. Brodsky, D. S. Hwang and I. Schmidt, Phys. Lett. B 530, 99 (2002) arXiv:hep-ph/0201296.

[98] J. C. Collins, L. Frankfurt and M. Strikman, Phys. Rev. D56, 2982 (1997) arXiv:hep-ph/9611433. 
[99] M. Diehl, T. Gousset, and B. Pire, arXiv:hep-ph/0003233].

[100] M. Ramsey-Musolf and M. B. Wise, arXiv:hep-ph/0201297.

[101] S. J. Brodsky, M. Diehl, P. Hoyer and S. Peigne, Phys. Lett. B 449, 306 (1999) arXiv:hep-ph/9812277.

[102] For an extensive review and further references see S. J. Brodsky, H. Pauli and S. S. Pinsky, Phys. Rept. 301, 299 (1998), arXiv:hep-ph/9705477.

[103] S. J. Brodsky and F. Schlumpf, Phys. Lett. B 329 (1994) 111.

[104] L. Okun and I. Yu. Kobzarev, JETP 161343 (1963).

[105] X. D. Ji, Phys. Rev. Lett. 78, 610 (1997) arXiv:hep-ph/9603249.

[106] X. D. Ji, Phys. Rev. D 58, 056003 (1998) arXiv:hep-ph/9710290.

[107] O. V. Teryaev, arXiv:hep-ph/9904376.

[108] V. M. Braun, A. Lenz, N. Mahnke and E. Stein, Phys. Rev. D 65, 074011 (2002) arXiv:hep-ph/0112085.

[109] S. J. Brodsky and D. S. Hwang, Nucl. Phys. B543, 239 (1999), arXiv:hep-ph/9806358.]

[110] C. R. Ji and H. M. Choi, Fizika B8, 321 (1999).

[111] K. Hornbostel, S. J. Brodsky, and H. C. Pauli, Phys. Rev. D41 3814 (1990).

[112] F. Antonuccio and S. Dalley, Phys. Lett. B348, 55 (1995); Phys. Lett. B376, 154 (1996); Nucl. Phys. B461, 275 (1996).

[113] H.-C. Pauli and S. J. Brodsky, Phys. Rev. D32 (1985) 1993 and 2001.

[114] S. Dalley, Nucl. Phys. Proc. Suppl. 90, 227 (2000) [arXiv:hep-ph/0007081]. See also M. Burkardt and S. K. Seal, Phys. Rev. D65, 034501 (2002).

[115] L. Del Debbio, M. Di Pierro, A. Dougall and C. Sachrajda, Nucl. Phys. Proc. Suppl. 83-84, 235 (2000) arXiv:hep-lat/9909147.

[116] V. Y. Petrov, M. V. Polyakov, R. Ruskov, C. Weiss and K. Goeke, Phys. Rev. D59, 114018 (1999) arXiv:hep-ph/9807229.

[117] D. Diakonov and V. Y. Petrov, arXiv:hep-ph/0009006.

[118] M. B. Hecht, C. D. Roberts and S. M. Schmidt, arXiv:nucl-th/0008049.

[119] L. L. Frankfurt and M. I. Strikman, Phys. Rept. 160, 235 (1988). 
[120] P. Jain, B. Pire and J. P. Ralston, Phys. Rept. 271, 67 (1996) arXiv:hepph/9511333.

[121] For a review of the experimental status of color transparency, see K. Griffioen, these proceedings.

[122] M. R. Adams et al. [E665 Collaboration], 470-GeV," Phys. Rev. Lett. 74, 1525 (1995).

[123] E. M. Aitala et al. [E791 Collaboration], Phys. Rev. Lett. 86, 4773 (2001) arXiv:hep-ex/0010044.

[124] A. S. Carroll et al., Phys. Rev. Lett. 61, 1698 (1988).

[125] Y. Mardor et al., Phys. Lett. B437, 257 (1998) arXiv:nucl-ex/9710002.

[126] A. Leksanov et al., Phys. Rev. Lett. 87, 212301 (2001) arXiv:hep-ex/0104039.

[127] G. R. Court et al., Phys. Rev. Lett. 57, 507 (1986).

[128] S. J. Brodsky and G. F. de Teramond, Phys. Rev. Lett. 60, 1924 (1988).

[129] G. F. de Teramond, R. Espinoza and M. Ortega-Rodriguez, Phys. Rev. D58, 034012 (1998) arXiv:hep-ph/9708202.

[130] J. P. Ralston and B. Pire, Phys. Rev. Lett. 57, 2330 (1986).

[131] S. J. Brodsky, E. Chudakov, P. Hoyer and J. M. Laget, Phys. Lett. B 498, 23 (2001) arXiv:hep-ph/0010343.

[132] G. Bertsch, S. J. Brodsky, A. S. Goldhaber, and J. F. Gunion, Phys. Rev. Lett. 47, 297 (1981).

[133] L. Frankfurt, G. A. Miller, and M. Strikman, Phys. Lett. B304, 1 (1993), arXiv:hep-ph/9305228.

[134] L. Frankfurt, G. A. Miller and M. Strikman, Found. Phys. 30, 533 (2000) arXiv:hep-ph/9907214.

[135] S. Brodsky, M. Diehl, P. Hoyer, and S. Peigne, in preparation.

[136] N. N. Nikolaev, W. Schafer and G. Schwiete, Phys. Rev. D 63, 014020 (2001) arXiv:hep-ph/0009038. N. Nikolaev and B. G. Zakharov, Z. Phys. C 53, 331 (1992).

[137] E. M. Aitala et al. [E791 Collaboration], Phys. Rev. Lett. 86, 4768 (2001) arXiv:hep-ex/0010043. 
[138] J. Gronberg et al. [CLEO Collaboration], Phys. Rev. D57, 33 (1998), hepex/9707031.

[139] V. M. Braun, D. Y. Ivanov, A. Schafer and L. Szymanowski, arXiv:hep$\mathrm{ph} / 0204191$.

[140] V. L. Chernyak, arXiv:hep-ph/0206144].

[141] I. P. Ivanov, N. N. Nikolaev, W. Schafer, B. G. Zakharov and V. R. Zoller, arXiv:hep-ph/0207045.

[142] C. W. Bauer, S. Fleming, D. Pirjol and I. W. Stewart, Phys. Rev. D 63, 114020 (2001) arXiv:hep-ph/0011336.

[143] M. Beneke, A. P. Chapovsky, M. Diehl and T. Feldmann, arXiv:hep$\mathrm{ph} / 0206152$. 